\title{
AN INVESTIGATION OF THE SPIN STRUCTURE OF THE PROTON IN DEEP INELASTIC SCATTERING OF POLARISED MUONS ON POLARISED PROTONS
}

\section{The European Muon Collaboration}

Aachen $^{1}$, CERN ${ }^{2}$, Freiburg ${ }^{3}$, MPI Heidelberg ${ }^{4}$, Lancaster $^{5}$, LAPP (Annecy) ${ }^{6}$, Liverpool $^{7}$, Marseille ${ }^{8}$, Mons $^{9}$, Oxford ${ }^{10}$, RAL (Chilton) ${ }^{11}$, Sheffield $^{12}$, Torino ${ }^{13}$, Uppsala ${ }^{14}$, Warsaw $^{15}$, Wuppertal ${ }^{16}$, Yale $^{17}$

$\mathrm{J} \mathrm{Ashman}^{12}$, B Badelek ${ }^{15 a)}$, G Baum ${ }^{17 b)}$, J Beaufays ${ }^{2}$, C P Bee ${ }^{7}$, C Benchouk ${ }^{8}$, I G Bird ${ }^{5 c)}$, S C Brown ${ }^{7 d)}, \mathrm{M} \mathrm{C}$ Caputo $^{17}, \mathrm{H} \mathrm{W} \mathrm{K} \mathrm{Cheung}{ }^{10 e)}$, J S Chima ${ }^{11 f)}$, J Ciborowski $^{15 a)}$, R Clifft ${ }^{11}, \mathrm{G}$ Coignet $^{6}, \mathrm{~F} \mathrm{Combley}^{12}, \mathrm{G} \mathrm{Court}^{7}$, G d'Agostini ${ }^{8}$, J Drees ${ }^{16}, \mathrm{M}$ Düren ${ }^{1}, \mathrm{~N}$ Dyce $^{5}, \mathrm{~A}$ W Edwards ${ }^{16 g}$ ), $\mathrm{M}$ Edwards ${ }^{11}$, T Ernst $^{3}$,

M I Ferrero ${ }^{13}$, D Francis ${ }^{7}$, E Gabathuler $^{7}$, R Gamet $^{7}$, V Gibson ${ }^{10 h}$, J Gillies ${ }^{10}$, $\mathrm{P}_{\text {Grafström }}{ }^{14 h)}$, K Hamacher ${ }^{16}$, D V Harrach ${ }^{4}$, P J Hayman ${ }^{7}$, J R Holt ${ }^{7}$, V W Hughes ${ }^{17}$, A Jacholkowska ${ }^{2 i)}$, T Jones ${ }^{7 k)}$, E M Kabuss ${ }^{3 c)}$, B Korzen ${ }^{16}$, U Krïner ${ }^{16}$, S Kullander ${ }^{14}$, U Landgraf ${ }^{3}$, D Lanske ${ }^{1}$, F Lettenström ${ }^{14}$, T Lindqvist ${ }^{14}$, J Loken ${ }^{10}, \mathrm{M} \mathrm{Matthews}^{7}, \mathrm{Y}$ Mizuno ${ }^{4}, \mathrm{~K} \mathrm{Mönig}^{16}, \mathrm{~F}_{\text {Montanet }}{ }^{8 h)}$, $\mathrm{E} \mathrm{Nagy}^{6 j}$, J Nassalski $^{15 l)}$, T Niinikoski ${ }^{2}$, P R Norton ${ }^{11}$, F G Oakham ${ }^{11 m)}$, R F Oppenheim ${ }^{17 n)}$, A M Osborne ${ }^{2}$, V Papavassiliou ${ }^{17}, \mathrm{~N} \mathrm{Pavel}^{16}, \mathrm{C}$ Peroni $^{13}, \mathrm{H}$ Peschel $^{16}$, R Piegaia ${ }^{17}$, B Pietrzyk ${ }^{8}$, U Pietrzyk ${ }^{160)}$, B Povh $^{4}$, P Renton ${ }^{10}, \mathrm{~J} \mathrm{M} \mathrm{Rieubland}^{2}$, A Rijllart ${ }^{2}$, K Rith ${ }^{3 c)}$,

E Rondio ${ }^{15 a)}$, L Ropelewski ${ }^{15 a)}$, D Salmon ${ }^{12 k)}$, A Sandacz ${ }^{15 l)}$, T Schröder ${ }^{3}$,

K P Schüler ${ }^{17}$, K Schultze ${ }^{1}$, T-A-Shibata ${ }^{4}$, T Sloan $^{5}$, A Staiano ${ }^{4 p)}$, H E Stier ${ }^{3}$, J Stock $^{3}$, G N Taylor ${ }^{10 q)}$, J C Thompson ${ }^{11}$, T Walcher ${ }^{4 r)}$, J $\operatorname{Toth}^{6 j}$,

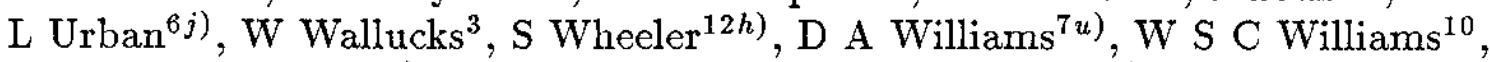
S J Wimpenny ${ }^{7 s}$, $\mathrm{R}$ Windmolders ${ }^{9}$, W J Womersley ${ }^{10 t)}$, K Ziemons ${ }^{1}$.

\section{Submitted to Nuclear Physics B}

\section{ABSTRACT}

The spin asymmetry in deep inelastic scattering of longitudinally polarised muons by longitudinally polarised protons has been measured in the range $0.01<x<0.7$. The spin dependent structure function $g_{1}(x)$ for the proton has been determined and, combining the data with earlier SLAC measurements, its integral over $x$ found to be $0.126 \pm 0.010$ (stat.) \pm 0.015 (syst.), in disagreement with the Ellis-Jaffe sum rule. Assuming the validity of the Bjorken sum rule, this result implies a significant negative value for the integral of $g_{1}$ for the neutron. These integrals lead to the conclusion, in the naive quark parton model, that the total quark spin constitutes a rather small fraction of the spin of the nucleon. Results are also presented on the asymmetries in inclusive hadron production which are consistent with the above picture.

For footnotes see next page 

a) University of Warsaw, Poland, partly supported by CPBP.01.06.
b) Permanent address, University of Bielefeld, FRG.
c) Now at MPI for Kernphysik, Heidelberg, FRG.
d) Now at TESA. S.A., Rennens, Switzerland.
e) Now at University of Colorado, Boulder, Colorado, USA.
f) Now at British Telecom, London, UK.
g) Now at Jet, Joint Undertaking, Abingdon, UK.
h) Now at CERN, Geneva, Switzerland.
i) Now at L.A.L., Orsay, France.
j) Permanent address, Central Research Institute for Physics of the Hungarian Academy of Science, Budapest, Hungary.
k) Now at R.A.L., Chilton, Didcot, UK.
1) Institute for Nuclear Studies, Warsaw, Poland, partly supported by CPBP.01.09.
m) Now at NRC, Ottawa, Canada.
n) Now at AT\&T, Bell Laboratories, Naperville, Illinois, USA.
o) Now at MPI for Neurologische Forschung, Köln, FRG.
p) Now at INFN, Torino, Italy.
q) Now at University of Melbourne, Parkville, Victoria, Australia.
r) Now at University of Mainz, Mainz, FRG.
s) Now at University of California, Riverside, USA.
t) Now at University of Florida, Gainesville, USA.
u) Now at UKAEA, Winfrith, Dorset, UK.

\section{Addresses}

1) 111 Physikalisches Institut A, Physikzentrum, RWTH, D-5100 Aachen, FRG.

2) CERN, CH-1211 Geneva 23, Switzerland.

3) Fakultät für Physik, Universität Freiburg, D-7800 Freiburg, FRG.

4) Max-Planck Institute for Kernphysik, Heidelberg, FRG.

5) Department of Physics, University of Lancaster, Lancaster LA1 4YB, UK.

6) Laboratoire d'Annecy-le-Vieux de Physique des Particules, F-BP110-74941, Annecyle-Vieux, France.

7) Department of Physics, University of Liverpool, Liverpool L69 3BX, UK.

8) Centre de Physique des Particules, Faculté des Sciences de Luminy, F-13288 Marseille, France.

9) Faculté des Sciences, Université de l'Etat à Mons, B-7000 Mons, Belgium. 
10) Nuclear Physics Laboratory, University of Oxford, Oxford OX1 3RH, UK.

11) Rutherford and Appleton Laboratory, Chilton, Didcot OX1 0QX, UK.

12) Department of Physics, University of Sheffield, Sheffield S3 7RH, UK.

13) Istituto di Fisica, Università di Torino, I-10125, Italy.

14) Department of Radiation Science, University of Uppsala, S-75121 Uppsala, Sweden.

15) Physics Institute, University of Warsaw and Institute for Nuclear Studies, 00681 Warsaw, Poland.

16) Fachbereich Physik, Universität Wuppertal, D-5600 Wuppertal, FRG.

17) Physics Department, Yale University, New Haven, Connecticut, USA. 


\section{INTRODUCTION}

Over the past two decades many experiments have studied the structure of the nucleon via deep inelastic scattering of charged leptons and neutrinos from unpolarised targets [1]. Such experiments have elucidated the quark-gluon structure of the nucleon and have shown that the quarks have half-integral spin. However, little information exists on how the spin of the nucleon is distributed among its constituents. Such information can be derived from a study of deep inelastic scattering of polarised leptons on polarised targets.

Prior to the present work only one such study had been carried out. This was the experiment at SLAC using polarised electrons scattered from a polarised proton target $[2,3,4]$. The experiment described here was designed with a similar objective in mind, but using a high energy beam of polarised positive muons from the CERN SPS with a target of polarised protons. This extends considerably the kinematic range of the observations and allows the spin structure of the proton to be studied in detail.

In this paper the measurements of the spin dependent asymmetry in the cross section for muon scattering are described, from which the spin dependent structure function of the proton $g_{1}(x)$ is deduced. Here $x$ is the fraction of the momentum of the proton carried by the struck quark. The integral of $g_{1}(x)$ over $x$ was used to test the Ellis-Jaffe sum rule [5] and to investigate the contribution of the spin of the quarks to the proton spin.

The final results presented here both extend and supersede those described in previous publications $[6,7,8]$.

\section{THE FORMALISM OF POLARISED DEEP INELASTIC SCATTERING}

The difference in the cross sections for deep inelastic scattering of muons polarised antiparallel and parallel to the spin of the target proton can be written in the single photon exchange approximation [9]

$$
{\frac{d^{2} \sigma}{d Q^{2} d \nu}}^{\uparrow \downarrow}-{\frac{d^{2} \sigma}{d Q^{2} d \nu}}^{\uparrow \uparrow}=\frac{4 \pi \alpha^{2}}{E^{2} Q^{2}}\left[M\left(E+E^{\prime} \cos \theta\right) G_{1}\left(Q^{2}, \nu\right)-Q^{2} G_{2}\left(Q^{2}, \nu\right)\right]
$$

where the variables are defined in Table 1 . The functions $G_{1}\left(Q^{2}, \nu\right)$ and $G_{2}\left(Q^{2}, \nu\right)$ are the spin dependent structure functions of the target nucleon. In the scaling limit as $Q^{2}$ and $\nu$ become large these structure functions are expected to become functions of $x$ only [10] so that

$$
\begin{aligned}
& M^{2} \nu G_{1}\left(Q^{2}, \nu\right) \rightarrow g_{1}(x), \\
& M \nu^{2} G_{2}\left(Q^{2}, \nu\right) \rightarrow g_{2}(x) .
\end{aligned}
$$

These structure functions can be obtained from experiments in which longitudinally polarised muons are scattered from longitudinally polarised target nucleons by measuring the 
asymmetry

$$
A=\frac{d \sigma^{\uparrow \downarrow}-d \sigma^{\uparrow \uparrow}}{d \sigma \uparrow \downarrow+d \sigma \uparrow \uparrow}
$$

This asymmetry is related through the optical theorem to the virtual photon asymmetries $A_{1}$ and $A_{2}$ by

$$
\begin{aligned}
A & =D\left(A_{1}+\eta A_{2}\right), \\
\text { where } A_{1} & =\frac{\sigma_{1 / 2}-\sigma_{3 / 2}}{\sigma_{1 / 2}+\sigma_{3 / 2}}, \\
A_{2} & =\frac{\sigma_{T L}}{\sigma_{T}}, \\
D & =\frac{y(2-y)}{y^{2}+2(1-y)(1+R)}, \\
\eta & =\frac{2(1-y)}{y(2-y)} \frac{\sqrt{Q^{2}}}{E} .
\end{aligned}
$$

Here $\sigma_{1 / 2}\left(\sigma_{3 / 2}\right)$ is the virtual photoabsorption cross section when the projection of the total angular momentum of the photon-nucleon system along the incident lepton direction is $1 / 2(3 / 2), \sigma_{T}=1 / 2\left(\sigma_{1 / 2}+\sigma_{3 / 2}\right)$ is the total transverse photoabsorption cross section and $\sigma_{T L}$ is a term arising from the interference between transverse and longitudinal amplitudes. The term $R$ in equation (7) is the ratio of the longitudinal to transverse photoabsorption cross sections and $D$ can be regarded as a depolarisation factor of the virtual photon.

The asymmetries $A_{1}$ and $A_{2}$ can be expressed in terms of the structure functions $g_{1}$ and $g_{2}[11]$ as

$$
\begin{aligned}
& A_{1}=\left(g_{1}-\gamma^{2} g_{2}\right) \frac{1}{F_{1}}, \\
& A_{2}=\gamma\left(g_{1}+g_{2}\right) \frac{1}{F_{1}},
\end{aligned}
$$

where $F_{1}$ is the spin independent structure function of the proton (the explicit $\left(Q^{2}, x\right)$ dependence of the structure functions has been omitted for brevity) and $\gamma=(2 M x / E y)^{1 / 2}$. Hence eliminating $g_{2}$ we obtain to first order in $\gamma$

$$
g_{1}=F_{1}\left(A_{1}+\gamma A_{2}\right) \text {. }
$$

Substituting for $A_{1}$ from (4) gives

$$
g_{1}=F_{1}\left(\frac{A}{D}+(\gamma-\eta) A_{2}\right)
$$

There are rigorous positivity limits on the asymmetries [12] i.e. $\left|A_{1}\right| \leq 1$ and $\left|A_{2}\right| \leq \sqrt{ } R$. Since $\gamma, \eta$ and $R$ are all small in the kinematic range of this experiment the term in $A_{2}$ may be neglected and

$$
A_{1} \simeq \frac{A}{D}
$$


so that

$$
g_{1} \simeq A_{1} F_{1}=\frac{A_{1} F_{2}}{2 x(1+R)},
$$

where $F_{2}$ is the second spin independent proton structure function. Neglecting $A_{2}$ in this way is equivalent to neglecting the contribution of $g_{2}$ which has been shown to have a negligible effect [13].

The structure function $g_{1}(x)$ is obtained as follows. The asymmetry $A$ (equation 3 ) is obtained from the experimental data, from which the virtual photon asymmetry $A_{1}$ is deduced via equation (11). The structure function $g_{1}(x)$ is then obtained from equation (12) using the known values of $F_{2}$ and $R$. The effect of neglecting $A_{2}$ is included in the systematic error, using the above mentioned limits for $A_{2}$.

\section{THEORETICAL MODELS}

By angular momentum conservation, a spin $\frac{1}{2}$ parton cannot absorb a photon when their two helicities are parallel. Hence in the quark-parton model (QPM), $\sigma_{1 / 2}\left(\sigma_{3 / 2}\right)$ can only receive contributions from partons whose helicities are parallel (antiparallel) to that of the nucleon. Hence it follows that

$$
A_{1}=\frac{\sigma_{1 / 2}-\sigma_{3 / 2}}{\sigma_{1 / 2}+\sigma_{3 / 2}}=\frac{\Sigma e_{i}^{2}\left(q_{i}^{+}(x)-q_{i}^{-}(x)\right)}{\Sigma e_{i}^{2}\left(q_{i}^{+}(x)+q_{i}^{-}(x)\right)},
$$

where $q_{i}^{+(-)}(x)$ is the distribution function for quarks of flavour $i$ and charge number $e_{i}$ whose helicity is parallel (antiparallel) to that of the nucleon. The sum is over all quark flavours $i$. In this model $F_{1}$ is given by

$$
F_{1}(x)=\frac{1}{2} \Sigma e_{i}^{2}\left(q_{i}^{+}(x)+q_{i}^{-}(x)\right) .
$$

Hence from equations (12) and (13), it follows that

$$
g_{1}(x)=\frac{1}{2} \Sigma e_{i}^{2}\left(q_{i}^{+}(x)-q_{i}^{-}(x)\right) .
$$

In the simple non-relativistic QPM [14] in which the proton consists of three valence quarks in an $\mathrm{SU}(6)$ symmetric wave function, $A_{1}^{p}=\frac{5}{9}$ and $A_{1}^{n}=0$ and are independent of $x$. Such a model clearly did not describe the SLAC data. Many models, mainly based on the QPM, were developed to predict the behaviour of the asymmetry $A_{1}$ (see [9], for a review). Models giving a good representation of the SLAC data were developed by Cheng and Fischbach [15], Callaway and Ellis [16], Carlitz and Kaur [17] and Schwinger [18]. Most of these incorporate the perturbative QCD prediction [19] that $A_{1}$ tends to unity as $x$ approaches unity and all except [18] are based on the QPM. These models predict roughly the same behaviour of $A_{1}$ and we choose arbitrarily to compare the data presented below with the Carlitz and Kaur model. 


\section{SUM RULES IN POLARISED DEEP INELASTIC SCATTERING}

A sum rule was developed by Bjorken [20] from light cone current algebra and with the assumption of quark structure for the hadronic electromagnetic and weak currents. It relates the integral over all $x$ of the difference of $g_{1}$ for the proton and neutron to the ratio of the axial vector to vector coupling constants in nucleon beta decay, $g_{A}$. In the scaling limit it can be written

$$
\int_{0}^{1}\left[g_{1}^{p}(x)-g_{1}^{n}(x)\right] d x=\frac{1}{6} g_{A}\left(1-\frac{\alpha_{s}}{\pi}\right)
$$

where the factor $\left(1-\alpha_{s} / \pi\right)$ arises from QCD radiative corrections [21]. This is a fundamental sum rule which represents a crucial test of the QPM [22].

Separate sum rules for the proton and the neutron were derived by Ellis and Jaffe [5] in a somewhat more model dependent approach. Assuming exact flavour SU(3) symmetry in the baryon-octet decays and that the net polarisation of the strange quark sea of the nucleon is zero, they derived

$$
\begin{aligned}
\int_{0}^{1} g_{1}^{p}(x) d x & =\frac{g_{A}}{12}\left[+1+\frac{5}{3} \frac{3 F / D-1}{F / D+1}\right] \\
\text { and } \int_{0}^{1} g_{1}^{n}(x) d x & =\frac{g_{A}}{12}\left[-1+\frac{5}{3} \frac{3 F / D-1}{F / D+1}\right],
\end{aligned}
$$

where $F$ and $D$ are the antisymmetric and symmetric SU(3) couplings [23]. Applying QCD radiative corrections to these yields [21]

$$
\int_{0}^{1} g_{1}^{p(n)}(x) d x=\frac{g_{A}}{12}\left[( \pm)\left(1-\frac{\alpha_{s}}{\pi}\right)+\frac{1}{3} \frac{3 F / D-1}{F / D+1}\left\{5-\left(1+4 \frac{33-8 f}{33-2 f}\right) \frac{\alpha_{s}}{\pi}\right\}\right],
$$

where $f$ is the number of quark flavours.

\section{EXPERIMENTAL PROCEDURE}

The experiment was performed in the M2 polarised muon beam at the CERN SPS using the EMC forward spectrometer [24] to detect the scattered muons and the fast forward hadrons produced by deep inelastic scattering in a longitudinally polarised target. For a fixed pion to muon energy ratio the muon beam was naturally longitudinally polarised since the muon produced in the rest frame of the parent pion has a fixed helicity. The polarised target [25] consisted of two cells filled with ammonia, separated by a gap, with the free protons in each cell polarised in opposite directions, parallel and antiparallel to the incident muon beam direction. The free proton asymmetry was obtained from the difference in the count rates of events reconstructed in each target cell. From this the asymmetry, $A_{1}(x)$ and the structure function $g_{1}(x)$ were deduced.

Figure 1 shows a schematic diagram of the apparatus. The trigger was provided by the scintillator hodoscopes $\mathrm{H} 1, \mathrm{H} 3$ and $\mathrm{H} 4$ which selected muons scattered through an angle 
greater than $\sim 3 / 4^{\circ}$. The scattered muon and forward hadrons were detected and measured in the system of multiwire proportional (P) and drift chambers (W) and their momenta analysed using a dipole field spectrometer magnet (FSM). Particles penetrating the $2.5 \mathrm{~m}$ thick steel absorber were labelled as muons. On receipt of a trigger the chambers were read out and the data written onto magnetic tape. These data were analysed using the EMC pattern recognition programme (PHOENIX) and the momentum analysis and vertex reconstruction programme (GEOM) to write data summary tapes. The apparatus used in this experiment (figure 1) is similar to that described previously [24] but was modified to run at the higher beam intensities required. To achieve this the drift chambers in the high background environment upstream of the magnet were replaced by proportional chambers (PV1, PV2). In addition further small proportional chambers (P0A-E), designed to work at high rates, were added in the beam region as well as the chambers $\mathrm{P} 4 / 5$. The latter provided extra information in the central region of $\mathrm{W} 4 / 5$ which had been found to deteriorate after prolonged exposure to radiation due to the deposition of silicon on the sense wires. With these modifications data were taken at beam intensities up to $4 \times 10^{7}$ per SPS pulse of 2 seconds duration, repeated every 14 seconds, i.e. approximately a factor 2 higher than previously.

Figure 2 shows a schematic diagram of the polarised target. The two cells, each of length $360 \mathrm{~mm}$ and volume 1 litre, were separated by a $220 \mathrm{~mm}$ gap. The target material was in the form of approximately spherical beads of solid ammonia each of volume $\sim 4 \mathrm{~mm}^{3}$, which allowed good cooling of the ammonia by the circulation of liquid helium through the spaces around the beads. The two cylindrical cells were positioned longitudinally along the beam line so that the same flux of incident muons passed through each. Very precise monitoring of the beam flux was then unnecessary since data were taken simultaneously for both directions of target polarisation.

The free protons in the ammonia were polarised in opposite directions in each cell by the method of dynamic nuclear polarisation. This method can be used for a small range of hydrogenous materials, of which ammonia has the highest hydrogen content. It requires that a dilute system of unpaired electron spins are introduced into the material. Such paramagnetic centres had been previously produced in the ammonia beads by irradiation with $25 \mathrm{MeV}$ electrons at a temperature of $90 \mathrm{~K}$, using the injection linear accelerator at the Bonn electron synchrotron. The electron spins from the paramagnetic centres become highly polarised when the material is placed in a strong magnetic field at a low temperature. This electron polarisation can be transferred to the protons by microwave irradiation at a frequency close to the electron spin resonance. The direction of the proton polarisation can be selected by making a small change $(\sim 0.6 \%)$ in the microwave frequency.

The magnetic field of $2.5 \mathrm{~T}$ was generated by a superconducting solenoid [26] of length $1.6 \mathrm{~m}$ and internal diameter $190 \mathrm{~mm}$, with its axis parallel to the muon beam direction to 
obtain longitudinally polarised protons. The field over the target volume was adjusted to be uniform to 1 part in $10^{4}$ with the aid of 12 trim coils. Such high uniformity was necessary to achieve resonance throughout the target volume. Each target cell was mounted in a separate conducting cavity of $150 \mathrm{~mm}$ diameter and supplied with microwave power at $\sim 70 \mathrm{GHz}$ from a separate microwave source, allowing independent control of the polarisation direction.

The target material was maintained at a temperature of about $0.5 \mathrm{~K}$, in the presence of input from the microwave sources, by a 2 watt ${ }^{3} \mathrm{He}-{ }^{4} \mathrm{He}$ dilution refrigerator [27]. The cooling system was common to both cells and so it was necessary to include a series of thin copper baffles and some microwave-absorbent material in the gap between the cells, to achieve isolation of the microwave power whilst allowing a free flow of the coolant.

The proton polarisation was measured continuously during data taking with a nuclear magnetic resonance system operating at a frequency of $106.3 \mathrm{MHz}$. This system had eight independent channels and sampled the polarisation with four coils, buried in the target material, in each cell. Calibration was carried out in the conventional way, using the calculable signal which is obtained when the proton spins in a known magnetic field are in thermal equilibrium with the solid lattice at a known temperature. The statistical uncertainty on the measurement of the NMR signal from a single coil was $\sim 1 \%$. The mean polarisation of a target cell was obtained by averaging the values from the four coils in that cell, which in general agreed to within $\sim 4 \%$. The overall error on this mean value arose from the polarisation non-uniformity together with uncertainties in the absolute determination of the calibration temperature and drifts in electronics. Thus the mean cell polarisation, which was typically between 0.75 and 0.80 , had an overall estimated uncertainty of \pm 0.05 .

In this experiment, which detected all final states inclusively, it was impossible to discriminate between scattering from free protons and from the unpolarised bound nucleons in the complex nuclei in the target. Thus the effective target polarisation was reduced by a factor $f$. The value of $f$, the dilution factor (see section 6.3), was maximised by using ammonia as the target material since it has the highest hydrogen content of the available materials. However, it suffers from the disadvantage of having a long polarisation reversal time ( $\sim 8$ hours). For this reason, it was not possible to reverse the polarisation directions more often than once per week without unacceptable loss of data taking time. A further problem was that the ${ }^{14} N$ nuclei in the ammonia, which have spin 1 , became slightly polarised [28], although this produced a negligible correction to the final results (see section 6.5 ).

The data were taken in 11 separate experimental runs at incident muon energies of 100 , 120 and $200 \mathrm{GeV}$. The apparatus acceptances from the two halves of the target differed by about $10 \%$. In order to correct for this and for the $\sim 1 \%$ difference in the target masses in each cell the polarisations were reversed once in each experimental run and the results before 
and after the reversal were averaged.

\section{DATA ANALYSIS}

\subsection{Introduction}

The free proton asymmetry A (equation 3) is extracted from the difference in counting rates of the events whose vertices were reconstructed in the two target cells. Figure 3 shows a reconstructed vertex distribution along the beam direction together with the cuts applied to define the events in each target cell. Using a Monte Carlo simulation of this distribution, it was shown that the events could be assigned to each target cell without ambiguity. The events reconstructed in between the target cells stem from interactions in the residual material (copper baffles and helium) in the gap and from the finite vertex resolution.

The measured event yields from the two target cells are

$$
\begin{aligned}
& N_{u}=n_{u} \quad b \quad a_{u} \quad \sigma_{0}\left(1-f P_{b} P_{u} A\right) \\
& \text { and } N_{d}=n_{d} \quad b \quad a_{d} \quad \sigma_{0}\left(1-f P_{b} P_{d} A\right) \text {, }
\end{aligned}
$$

where the subscript $u(d)$ refers to the upstream (downstream) target half, $n$ is the number of target nucleons, $b$ the beam flux, $a$ the apparatus acceptance, $\sigma_{0}$ the unpolarised cross section, $f$ the fraction of the event yield from the polarised protons in the target, $P_{b}, P_{u}\left(P_{d}\right)$ the beam and target polarisations, respectively. The phase space cuts on the beam ensured that the beam flux was the same for both target halves. The sign of the polarisation of both target and incident muon is defined to be positive when parallel to the incident positive muon beam direction. With this definition $P_{b}$ was always negative and $P_{u}$ and $P_{d}$ were of opposite sign. For an experimental run where $P_{u}$ was initially positive and $P_{d}$ negative the measured asymmetries are

$$
A_{m}=\frac{N_{u}-N_{d}}{N_{u}+N_{d}} \text { and } A_{m}^{\prime}=\frac{N_{d}^{\prime}-N_{u}^{\prime}}{N_{d}^{\prime}+N_{u}^{\prime}}
$$

where the primed (unprimed) quantities refer to the quantities measured after (before) the polarisation reversal during the experimental run. The free proton asymmetry is related to the measured asymmetries by

$$
\overline{A_{m}}=\frac{1}{2}\left(A_{m}+A_{m}^{\prime}\right)=f P_{B} P_{T} A \simeq f P_{B} P_{T} D A_{1}
$$

where $P_{B}=\left|P_{b}\right|$ and $P_{T}=\left(\left|P_{u}\right|+\left|P_{d}\right|+\left|P_{u}^{\prime}\right|+\left|P_{d}^{\prime}\right|\right) / 4$ is the average target polarisation. Values of $\overline{A_{m}}$ as a function of $x$ for the total data sample are given in table 5 . The values are always less than $\sim 0.02$, so it was vital to control all possible sources of systematic false asymmetries to much better than this figure. This was the reason for having the split target design, since the uncertainty on the measurement of the muon flux through the target was of the same order as the measured asymmetry. All false asymmetries cancel from equation (20) 
except those due to time dependent acceptance changes. Such an effect would occur only if the ratio of the upstream to downstream acceptance ratios before and after the polarisation reversal,

$$
K=\frac{a_{u} / a_{d}}{a_{u}^{\prime} / a_{d}^{\prime}},
$$

were different from unity. This would produce a false asymmetry which would induce a systematic error in the results. This will be discussed later.

\subsection{The Beam Polarisation}

In the laboratory frame the muon polarisation is given by

$$
P_{b}=\mp \frac{\lambda-m_{\mu}^{2} / m_{\pi}^{2}(1-\lambda)}{\lambda+m_{\mu}^{2} / m_{\pi}^{2}(1-\lambda)},
$$

where

$$
\lambda=\frac{E_{\mu}-E_{\min }}{E_{\pi}-E_{\min }}
$$

with $m_{\mu}, m_{\pi}$ the muon and pion masses, $E_{\mu}, E_{\pi}$ their energies in the laboratory frame and $E_{\min }=\left(m_{\mu}^{2} / m_{\pi}^{2}\right) E_{\pi}$ is the minimum allowed muon energy in the laboratory frame. The negative (positive) sign is for positive (negative) muons. The beam polarisation was computed by averaging equation (22) over the beam phase space in a Monte Carlo simulation of the beam [29]. Previous measurements of the beam polarisation [30] agreed with the predictions of this Monte Carlo simulation within measurement errors of 10-15\%. Table 2 shows the computed beam polarisation for each of the three settings used in this experiment. The quoted errors arise from the uncertainties in the beam phase space and in the contamination of the parent $\pi$ beam by $K$ mesons ( $18 \pm 9 \%$ ).

\subsection{The Dilution Factor}

The dilution factor $f$ is the fraction of the events arising from scattering by the polarised protons in the target. To a first approximation $f$ is $3 / 17$ for the ammonia $\left(\mathrm{NH}_{3}\right)$ target representing 3 free protons out of 17 nucleons per molecule. However, several other effects must be taken into account. Firstly the neutron and proton cross sections are not the same. Parameterising the available data $[31,32]$ gives

$$
\frac{\sigma_{n}}{\sigma_{p}}=0.92-0.883 x
$$

with an uncertainty of $\sim \pm .05$ independently of $x$. Secondly, the cross section for bound nucleons is not the same as that for free nucleons [33], the "EMC effect". Parameterising the data for carbon [34], which is assumed to be similar to nitrogen,

$$
h(x)=\frac{\sigma(\text { bound })}{\sigma(\text { free })}=1.06-0.30 x-0.45 e^{-44 x}
$$


The uncertainty in this ratio was taken to be either 0.03 or $0.5(1-h(x))$ whichever is the larger. Thirdly, other material (helium and copper) within the target cells contributed $\sim 11 \%$ of the rate from the ammonia, with an estimated error of $20 \%$ of its value. Fourthly, events originating from unpolarised material outside the target cell contaminate the sample due to resolution smearing. This was estimated by a Monte Carlo simulation to be $(6.6 \pm 0.7) \%$ with an estimated systematic error of $3 \%$.

Taking all these effects into account the dilution factor becomes

$$
f=\frac{3}{3+h(x)\left(8.84+8.44 \frac{\sigma_{n}}{\sigma_{p}}\right)}
$$

\subsection{The Virtual Photon Depolarisation Factor D}

The factor $D$ is defined in equation 7. To compute it the values of $R=\sigma_{L} / \sigma_{T}$ were calculated using perturbative QCD [35]. These represent the measurements quite well [1] within the rather large errors. Accordingly an error equal to $50 \%$ of the value calculated from QCD was assigned to $R$. A parameterisation of $R$ calculated in this way at the mean $Q^{2}$ value in each $x$ bin for this experiment is

$$
R=0.0122 /(x+0.041)^{1.096} .
$$

\subsection{The Corrections for Radiative Effects and the Nitrogen Polarisation}

The quantities of interest, $A_{1}$ and $g_{1}$, (equations 11 and 12) are defined in the one photon exchange approximation, while the measured quantities contain contributions from higher order processes and must therefore be corrected. The formulae of Mo and Tsai [36] are used for these radiative corrections. Although the formulae are strictly valid only for spin averaged cross sections the results are very similar to those of a more exact treatment of Kukhto and Shumeiko [37]. The corrections also included allowance for the slight polarisation of the nitrogen nuclei. In detail the corrections were applied as follows.

The measured cross section $\sigma_{m}$ can be written as

$$
\sigma_{m}(x, y)=B_{k} \sigma_{1 \gamma}+\sigma_{i n e l}^{R}+\sigma_{e l}^{R}
$$

where $\sigma_{1 \gamma}$ is the one photon exchange cross section, $B_{k}$ is a correction factor to the virtual photon flux for the vacuum polarisation, vertex graphs etc. and $\sigma_{i n e l}^{R}\left(\sigma_{e l}^{R}\right)$ is the contribution to the cross section in a given $x, y$ bin from the inelastic (elastic) radiative tails:

$$
\sigma_{\text {inel(el) }}^{R}(x, y)=\int r\left(x^{\prime}, y^{\prime}, x, y\right) \sigma_{\text {inel }(e l)}^{R}\left(x^{\prime}, y^{\prime}\right) d x^{\prime} d y^{\prime}
$$


Here $r\left(x^{\prime}, y^{\prime}, x, y\right)$ is the probability that an event at $x^{\prime}, y^{\prime}$ appears, after radiating one or more photons, in the bin $x, y$.

The measured asymmetry can then be written as

$$
\begin{aligned}
A_{m} & =f P_{B} P_{T} D A_{1}\left(\frac{B_{k}+T_{1}+T_{2}+\frac{P_{T}^{N}}{P_{T}}\left(T_{3}+T_{4}+T_{5}+T_{6}\right)}{1+\left(T_{7}+T_{8}\right) f}\right) \\
& =\frac{f P_{B} P_{T} D A_{1}}{1+R_{c}}
\end{aligned}
$$

where $R_{c}$ is the overall correction, $P_{T}^{N}$ is the nitrogen polarisation (13\% of the proton polarisation $\left.P_{T}[28]\right)$ and the different terms $T_{i}$ are:

$T_{1}$ : radiated asymmetry from the proton inelastic tail

$$
T_{1}=\frac{1}{D A_{1} \sigma_{1 \gamma}} \int r\left(x^{\prime}, y^{\prime}, x, y\right) D\left(y^{\prime}\right) A_{1}\left(x^{\prime}\right) \sigma_{\text {inel }}\left(x^{\prime}, y^{\prime}\right) d x^{\prime} d y^{\prime},
$$

with $A_{1}$ taken from a fit to the data.

$T_{2}$ : radiative asymmetry from the proton elastic tail. It is given by an expression identical to (29) but with the elastic asymmetry $A_{e l}$ (arising from the interference of $G_{M}$ and $G_{E}$, which have been determined to have the same sign [38]) substituted for $A_{1}$ :

$$
A_{e l}=D_{e l}\left(1+\eta \sqrt{R_{e l}}\right)
$$

with

$$
D_{e l}=\frac{y(2-y)}{y^{2}+2(1-y)\left(1+R_{e l}\right)}
$$

and

$$
R_{e l}=\frac{G_{E}^{2}}{\tau G_{M}^{2}}
$$

where $G_{M}$ and $G_{E}$ are the electric and magnetic form factors of the proton and $\tau=Q^{2} / 4 M^{2}$.

$T_{3}$ : correction for the asymmetry from the polarised nitrogen

$$
T_{3}=B_{k} \frac{\sigma^{N} A_{1}^{N}}{3 \sigma^{p} A_{1}^{p}},
$$

where $\sigma^{N}, \sigma^{p}, A_{1}^{N}$ and $A_{1}^{p}$ are the cross sections and asymmetries for nitrogen and proton, respectively. The asymmetry $A_{1}^{N}$ was computed using the shell model of the nucleus in which the nitrogen nucleus consists of a spin 0 core of 6 protons and 6 neutrons plus an odd proton and neutron each in a $p^{\frac{1}{2}}$ state so that the ground state has spin 1 . Writing down the nuclear wave functions shows that each odd nucleon is twice as likely to have its spin opposite to the nuclear spin than parallel to it. Such a calculation predicts the 
static magnetic moments of ${ }^{14} \mathrm{~N}$ to within $10 \%$ of the measured value. Thus neglecting the asymmetry from the odd neutron and assuming that the bound and free proton asymmetries are the same $A_{1}^{N} \sim-\frac{1}{3} \frac{\sigma^{p}}{\sigma^{N}} A_{1}^{p}$, so that $T_{3} \sim-B_{k} / 9$. On multiplying this by the ratio $P_{T}^{N} / P_{T}$, the polarisation of the nitrogen nucleus contributes a correction $\sim 1.5 \%$ to the free proton asymmetry.

$T_{4}$ : Correction due to the inelastic radiative tail from the polarised proton in the nitrogen (as in equation 29, with $A_{1}^{N}$ substituted for $A_{1}$ ).

$T_{5}$ : Correction for the quasi-elastic radiative tail from nitrogen.

$T_{6}$ : Correction for the coherent radiative tail from nitrogen.

$T_{7}$ : Total radiative correction for unpolarised protons.

$T_{8}$ : Total radiative correction for unpolarised nitrogen. Here the single nucleon cross section for carbon was used, which should be similar to that for nitrogen.

Fig. 4 shows the contribution of the various sources to the radiative correction. The dash-dotted curve, labelled "Polarised Proton Correction", is obtained from $B_{k}+T_{1}+T_{2}-1$ (equation 28). It shows the effect in the numerator of the asymmetry arising from radiative smearing in elastic and inelastic scattering together with the effects of the vacuum polarisation and vertex corrections. The term $T_{2}$ from elastic scattering is everywhere small. The dashed curve, labelled "Polarised Nitrogen Correction", is obtained from $\frac{P_{T}^{N}}{P_{T}}\left(T_{3}+T_{4}+T_{5}+T_{6}\right)$ which is dominated by $T_{3}$. The correction is rather small ( $<2 \%$ everywhere). The solid curve, labelled "Unpolarised Correction" shows the term $f\left(T_{7}+T_{8}\right)$ which represents the correction to the unpolarised cross sections in the denominator of the asymmetry. The values are dominated by the nitrogen contribution $\left(T_{8}\right)$ which included quasi-elastic and coherent elastic radiative scattering as well as the contribution from radiative inelastic scattering and vacuum polarisation and vertex effects. The unpolarised correction gives the largest contribution to the radiative corrections.

The total radiative correction to the measured value of $A_{1}$ (the term $R_{\mathrm{c}}$ in equation 28 ) is shown as a function of $x$ in figure 5. Also shown are the variation of the depolarisation factor $D$, the dilution factor $f$, and the mean value of $y$.

Electroweak effects were also studied but were found to be negligible in the $Q^{2}$ range of this experiment.

\section{RESULTS}

\subsection{The virtual Photon Asymmetry $\mathbf{A}_{1}$}

The cuts applied to the data are given in table 3 and the numbers of events surviving these cuts in table 4 together with other details of each experimental run. The virtual photon 
asymmetry $A_{1}$ was calculated for each experimental run on a grid of $11 x$ and $15 Q^{2}$ bins. The data were then averaged in different ways. Table 5 and figure 6 show the values of $A_{1}$ as a function of $x$ averaged over $Q^{2}$. The systematic errors shown in table 5 are discussed below. The values of $\chi^{2}$ to the mean of each $x$ point for 10 degrees of freedom (11 runs) are also given. These show approximately a statistical distribution which is evidence that systematic errors due to false asymmetries are smaller than the statistical errors, provided that they do not always contribute in the same direction. A parameterisation of the data in fig. 6 is

$$
A_{1}=1.025 x^{0.12}\left(1-e^{-2.7 x}\right) \text {. }
$$

The earlier data from SLAC $[2,3]$ are also shown in fig. 6. The agreement between these data and the data presented here is good in the region of overlap. The new measurements extend the range down to lower values of $x$. The solid smooth curve in fig. 6 shows the predictions of the model of Carlitz and Kaur [17] based on the conventional quark parton model. This model gives a good representation of the data for $x \geq 0.2$ but fails to represent the new data at lower values of $x$. A recent modification of this model, allowing the $u$ and $d$ quarks to have different masses, obtained good agreement with the data over the whole $x$ range [39]. Predictions of the behaviour of $A_{1}$ with $x$ were also made using the Fire String Model [40]. These predictions are in good agreement with the data in fig. 6 .

Fig. 7 shows the $Q^{2}$ dependence of $A_{1}$ in three $x$ ranges together with the older SLAC data in the deep inelastic region [2,3] and also in the resonance region [4] in which a $W$ cut (W $>1.31 \mathrm{GeV}$ ) has been applied to exclude the $\Delta_{33}$ (1236) resonance where the asymmetry is observed to be negative. This figure shows that there is no strong $Q^{2}$ dependence in the data. However, the predicted scaling violations due to QCD effects [41] are much smaller than the precision of the data. This negligible $Q^{2}$ dependence of $A_{1}$ at fixed $x$ allows us to combine the data taken with different beam energies in the same $x$ bin.

\subsection{The systematic Errors on $A_{1}$}

The systematic errors on $A_{1}$, shown in table 5 , were evaluated from each of the individual sources shown in table 6 . The value of $R$ used to compute the depolarisation factor was taken from a QCD calculation [35] with a $50 \%$ uncertainty as explained above (section 6.4). The change in the value of $A_{1}$ as the computed value of $R$ is changed by $50 \%$ are shown in the second column of table 6 and this is taken as the uncertainty due to $R$. Similarly the uncertainty due to the neglect of $A_{2}$ in equation (4) was obtained by recalculating $A_{1}$ assuming $A_{2}$ can be anywhere within the limits $-\sqrt{R} \leq A_{2} \leq \sqrt{R}$, set by positivity requirements [12]. Taking $R$ from the QCD calculation, as above, the changes in $A_{1}$ produced by neglecting $A_{2}$ in this way are shown in the third column of table 6 .

The dilution factor $f$ (equation 25) suffers from uncertainties as described in section 6.3. The total error on $A_{1}$ induced by these errors on $f$ are shown in the fifth column of table 6 . 
The uncertainties in the radiative corrections reflect both theoretical uncertainties and those due to approximations made when applying the corrections to the data. The uncertainty assigned was $15 \%$ of the correction or $1 \%$ of the measured value of $A_{1}$, whichever was larger. Due to the smallness of the correction itself, this is a relatively unimportant source of error for $A_{1}$. It is shown in column 6 of table 6 .

The uncertainty labelled $K$ in column 4 of table 6 is an estimate of the error arising from possible false asymmetries due to time dependent changes in the ratio of the upstream to downstream acceptances $\left(a_{u} / a_{d}\right)$. This is quantified by $K$, the term defined in equation (21). If $K$ is not exactly unity, then the measured asymmetry (equation 20) becomes, to first order in $K-1$,

$$
A_{1}=\frac{1}{f P_{B} P_{T} D}\left[\frac{1}{2}\left(A_{m}+A_{m}^{\prime}\right) \pm \frac{K-1}{4}\right]
$$

where the $+(-)$ sign is for periods of type $1(2)$, i.e. those in which the initial target configuration is $-/+$ i.e. $P_{u}<0, P_{d}>0\left(+/-\right.$ i.e. $\left.P_{u}>0, P_{d}<0\right)$. Figure 8 shows the values of $A_{1}$ as a function of $x$ for the data averaged over the seven periods of type 1 and over the four periods of type 2. The fact that the data for type 1 periods tend to have larger values of $A_{1}$ than those for type 2 shows that $K$ is not exactly unity. The values of $K$ in each $x$ bin required to reconcile the differences in fig. 8 was determined using equation (36). These values turned out to be constant within errors, i.e. independent of $x$ with a mean value of $0.990 \pm .005$. In doing this the mean value of $K$ in each $x$ bin was assumed to be the same for the 7 type 1 periods as the 4 type 2 periods. An approximately time independent value of $K$ is expected since the ratio $a_{u} / a_{d}$ tended to increase uniformly with time due to the radiation damage to the chambers in the beam region.

Since seven periods were of type 1 and four of type 2 , there is a partial cancellation of the false asymmetry term $\pm(K-1) / 4$ in equation (36) when all eleven periods are combined together. The above value of $K$ of $0.990 \pm 0.005$ for each period becomes an effective $K_{\text {tot }}$ of $0.998 \pm 0.001$ when all periods are combined together. To check this result the data were split into two subsamples for one of which time dependent changes in the ratio $a_{u} / a_{d}$ were expected to be much smaller than for the other. Thus the asymmetry $A_{1}$ was determined for the subsample of events in which the scattered muon passed outside the radius of $\mathrm{P} 4 / 5$. The values of $A_{1}$ for this subsample were consistent within the errors for type 1 and type 2 periods. This was expected since no change in chamber efficiencies outside this radius could be detected, and hence it can be assumed that the value of $K$ for these events is close to unity. Labelling $A_{1}$ for this subsample as $A_{\text {out }}$, and for the total event sample as $A_{\text {tot }}$, we then have

$$
K_{\text {tot }}-1=4 f P_{B} P_{T} D\left(A_{\text {out }}-A_{\text {tot }}\right)
$$

The points derived from the above equation are shown in figure 9 as a function of $x$. The values of $K_{\text {tot }}$ are everywhere consistent with unity and have an average of $1.003 \pm 0.002$ in 
reasonable agreement with the previous value of $0.998 \pm 0.001$. Since both these values are consistent with unity, it was decided to take $K_{\text {tot }}=1.000 \pm 0.003$, constant and independent of $x$. Hence no systematic correction was applied to the values of $A_{1}$, but the above uncertainty was translated into a systematic error on $A_{1}$, where values are shown in column four of table 6.

As a consistency check on the above analysis events were selected which contained an identified hadron. For this sample the radiative corrections were small since all the effects concerning the elastic radiative tail disappear on demanding a hadron. In addition for such events $a_{u} / a_{d} \sim 0.8$ averaged over $x$ compared to about 1.1 for the total inclusive sample. Thus any time dependent changes in $a_{u} / a_{d}$ would be expected to have a different effect between the two samples. Particles were identified as hadrons and not electrons by demanding that less than $85 \%$ of their total energy was deposited in the upstream electromagnetic part of the calorimeter ( $H_{2}$ in fig. 1). Figure 10 shows the variation of $A_{1}$ as a function of $x$ for events with accompanying hadrons compared to the values from the total sample. There is a good consistency between the two sets of data, illustrating that the radiative effects had been correctly calculated and residual false asymmetries were small compared to the errors.

The data were split into two different subsamples in many other ways. None of these gave a mean value of $K$ which was significantly different from unity.

\subsection{Semi-inclusive Asymmetries in the Final State Hadrons}

Spin asymmetries for positive and negative hadron production were also measured. Here the asymmetries are given by

$$
A^{ \pm}=\frac{\left(\frac{d \sigma^{ \pm}}{d z}\right)_{\frac{1}{2}}-\left(\frac{d \sigma^{ \pm}}{d z}\right)_{\frac{3}{2}}}{\left(\frac{d \sigma^{ \pm}}{d z}\right)_{\frac{1}{2}}+\left(\frac{d \sigma^{ \pm}}{d z}\right)_{\frac{3}{2}}}
$$

where again the subscripts refer to the projection of the total angular momentum of the virtual photon-proton system along the incident lepton direction and the $+(-)$ signs refer to positive (negative) hadrons. In the naïve quark parton model $A^{+}$is expected to be larger than $A^{-}$. This can be understood as follows. From helicity conservation the cross section for quark scattering $\sigma_{3 / 2}^{q}$ is zero and $u(d)$ quarks fragment more readily to $\pi^{+}\left(\pi^{-}\right)$mesons, particularly at higher $z$ [42] where $z=E_{\pi} / \nu$ with $E_{\pi}$ the pion energy. Thus if the $u(d)$ quarks are polarised parallel (antiparallel) to the proton spin as expected in the naive quark parton model $A^{+}$should be larger than $A^{-}$at higher $z$. Detailed calculations based on this model were made by Heimann [43].

In order to maximise the difference between $A^{+}$and $A^{-} z$ should be as large as possible. The analysis was performed with $z>z_{0}$ with $z_{0}=0.1$ as a compromise between sufficient 
statistical accuracy and maximising the expected differences between $A^{+}$and $A^{-}$. The analysis procedure is identical to that for the inclusive asymmetries described above except for the calculation of the dilution factors. This stems from the different probabilities for a proton and neutron in the target to yield final state $\pi^{+}$and $\pi^{-}$mesons. These dilution factors were computed from the quark parton model using the quark distribution function taken from [44] and the parameterisations of the favoured and unfavoured fragmentation functions taken from [42]. The results are shown in table 7 and fig. 11.

It can be seen from fig. 11 that both $A^{+}$and $A^{-}$rise at large values of $x$. This is to be expected from the observed behaviour of the inclusive asymmetry (section 7.1). However, the values of $A^{+}$tend to be larger than those of $A^{-}$, consistent with the expectations of the naive quark parton model.

\section{THE STRUCTURE FUNCTION $\mathbf{g}_{1}^{p}$}

The structure function $g_{1}^{p}$ was determined at a fixed $Q^{2}=10.7 \mathrm{GeV}^{2}$, the mean $Q^{2}$ of the data, using equation (12) and the values of $A_{1}$ from table 5. As shown in this table, the mean $Q^{2}$ for these values varies considerably with $x$, but (from section 7.1) there is no change of $A_{1}$ with $Q^{2}$ at fixed $x$ within the errors. The values of $F_{2}$ were taken from a parameterisation of the EMC hydrogen data [45] adjusted from the value of $R=0$ which was assumed in this parameterisation, to $R$ calculated from QCD. Table 8 and figure 12 show the values obtained for $g_{1}^{p}$ together with the systematic uncertainties described in section 7 . The total normalisation uncertainty of $14 \%$ arises from $9.6 \%$ due to the uncertainty in the beam and target polarisations and an assumed $10 \%$ due to the uncertainty in $F_{2}$.

The value of $F_{2} /(1+R)$ for $x<0.03$ was taken to be constant as expected from Regge theory [46] and as confirmed experimentally up to $Q^{2}=7 \mathrm{GeV}^{2}$ [47]. The data in figure 12 tend to be constant (within errors) for $x<0.2$ as predicted from simple Regge theory $[46,48]$.

\section{THE INTEGRAL OF $g_{1}^{\text {P OVER } x}$}

\subsection{The EMC Data Alone}

In integrating $g_{1}^{p}$ over $x$ the values of $A_{1}$ were assumed constant over each $x$ bin, but the function $F_{2} / 2 x(1+R)$ was integrated numerically for each bin because of its rapid variation for $x>0.3$. Figure 13 shows the values of this integral from the low edge of each bin to $x=1$, plotted against the low edge of the bin, together with the data from SLAC [2,3]. The inner and outer error bars are the statistical and total errors. It should be noted that the errors are cumulative i.e. each error contains the contribution from all the previous points at higher $x$. The normalisation error is included in the total error. The smooth curve is the integral obtained by using the parameterisation of $A_{1}$ (equation 35) which was used to estimate the contributions from the regions in $x$ not covered by the data i.e. $x<0.01$ and $x>0.7$. 
It can be seen that contributions from the lower $x$ bins are small and the integral converges well. The values of the integral shown in fig. 13 were obtained using a parameterisation of the EMC measurements of $F_{2}$ for the proton [45]. Recently, some differences between the various measurements of $F_{2}$ have been highlighted [49]. To test the sensitivity to $F_{2}$ the integral was evaluated using the different available data on $F_{2}$. The results in the measurement region $0.01<x<0.7$ are shown in table 9 at $Q^{2}=10.7 \mathrm{GeV}^{2}$, the mean $Q^{2}$ of the data. The first two values of $F_{2}$ are the EMC proton [45] and iron data [50] where in the latter a correction has been made for nuclear effects on the nucleon structure function $[33,34]$ and for the ratio of $\sigma_{n} / \sigma_{p}$ from the equation (23). The entry labelled DFLM in table 9 uses the value of $F_{2}$ computed from the most recent parameterisation of the neutrino structure functions [44] which are based on data down to $x=0.015$. The entry labelled BCDMS is from a parameterisation of the data given in [51]. To extrapolate the latter data set below their measurement region $(x<0.06)$ the assumption was made that $F_{2} /(1+R)$ approaches a constant below $x=0.06$ as discussed in section 8 .

Taking the mean of the values in table 9 gives

$$
\int_{0.01}^{0.7} g_{1}^{p} d x=0.120 \pm 0.013 \text { (stat.) }
$$

with an uncertainty due to $F_{2}=5.6 \%$ which is taken from the standard deviation of the values in table 9 .

The contributions outside the measured region were obtained from the parameterisation of $A_{1}$ (equation 35 ) and these give

$$
\int_{0.7}^{1} g_{1}^{p} d x=0.001 \quad \text { and } \quad \int_{0}^{0.01} g_{1}^{p}=0.002
$$

In the latter we assume that both $g_{1}$ and $F_{2} /(1+R)$ are well behaved, i.e. remain approximately constant as $x$ approaches zero. For $g_{1}$ this is compatible with the data (fig. 12) and it has been shown to be true for $F_{2}$ up to $Q^{2} \sim 7 \mathrm{GeV}^{2}$ in a recent experiment [47]. We assign errors equal to the values in equation (39).

The systematic errors affect the values in all the bins in the same way. The contribution to the total uncertainty from each separate source is estimated by recalculating the integral after increasing or decreasing all the points simultaneously by the corresponding systematic error. Table 10 summarises the results together with the global uncertainty which is obtained from the quadrature sum of the individual contributions. Thus from the asymmetry measurements presented here the integral becomes

$$
\int_{0}^{1} g_{1}^{p} d x=0.123 \pm 0.013 \pm 0.019
$$


where the first is the statistical and the second the systematic error.

\subsection{Combination of the EMC and SLAC Data [2,3]}

Since we have already shown (fig. 7) that there is no indication of a $Q^{2}$ dependence of $A_{1}$, over the range covered by the EMC and SLAC data, it is reasonable to combine the results to achieve higher accuracy. Averaging over the different sources of $F_{2}$, as above, the SLAC data give

$$
\int_{0.1}^{0.7} g_{1}^{p} d x=0.094 \pm 0.008 \pm 0.014
$$

and in the same region $(0.1<x<0.7)$ the EMC data give

$$
\int_{0.1}^{0.7} g_{1}^{p} d x=0.090 \pm 0.010 \pm 0.011
$$

where the contribution to the systematic error from the uncertainty in $F_{2}$ has been excluded.

The systematic errors in the two results have different origins, being dominated by the uncertainty due to possible false asymmetries from acceptance effects in the EMC case and by the value of $R$ in the SLAC case. Therefore the systematic errors can be combined as if they were statistical, giving

$$
\int_{0.1}^{0.7} g_{1}^{p} d x=0.092 \pm 0.006 \pm 0.010
$$

where a further $5 \%$ contribution has now been added for the uncertainty in $F_{2}$ in this $x$ range. In addition the EMC data alone gives

$$
\int_{0.01}^{0.1} g_{1}^{p} d x=0.030 \pm 0.008 \pm 0.007
$$

where the systematic error includes the uncertainty in $F_{2}$. In combining (43) and (44), care must be taken regarding the correlation in the uncertainties for EMC data in the low and high $x$ ranges. If the systematic errors in (43) and (44) were uncorrelated, they should be added in quadrature whereas if they were correlated they should be added linearly. Since (43) was obtained with approximately equal contributions from SLAC and EMC, the mean of the values of the two approaches is taken. Adding the contributions from extrapolating into the unmeasured regions gives

$$
\int_{0}^{1} g_{1}^{p} d x=0.126 \pm 0.010 \pm 0.015 .
$$

The value expected for this integral from the Ellis-Jaffe sum rule (equation 17) is $0.189 \pm 0.005$ using the current values of $\mathrm{F} / \mathrm{D}=0.631 \pm 0.018[52], g_{A}=1.254 \pm 0.006$ and $\alpha_{s}=0.27 \pm .02$ at $Q^{2}=10.7 \mathrm{GeV}^{2}$. The measured value is inconsistent with this prediction. 


\section{DISCUSSION OF THE RESULTS AND CONCLUSIONS}

The QCD corrected parton model expression for the integral of $g_{1}^{p}$ can be written [53]

$$
\Gamma_{p}=\int_{0}^{1} g_{1}^{p} d x=\frac{1}{12}\left\{\left[1-\frac{\alpha_{s}}{\pi}\right]\left[a_{3}+\frac{1}{\sqrt{3}} a_{8}\right]+2 \sqrt{\frac{2}{3}}\left[1-\frac{33-8 f}{33-2 f} \frac{\alpha_{s}}{\pi}\right] a_{0}\right\}
$$

where the $a_{j}$ are directly related to the proton matrix elements of the nonet of axial vector currents $A_{j}^{\mu}=\bar{\Psi} \gamma^{\mu} \gamma_{5} \frac{\lambda_{j}}{2} \Psi, j=0,1, \ldots 8$ by $\left\langle P, S\left|A_{j}^{\mu}\right| P, S\right\rangle=2 M a_{j} S^{\mu}$ where $S^{\mu}$ is the covariant spin vector of the proton.

From isospin invariance it follows that $[20]$

$$
a_{3}=g_{A}=F+D=1.254 \pm .006
$$

Furthermore, if $S U(3)_{F}$ is a good symmetry for describing the $\beta$ decays of the octet of hyperons [5]

$$
a_{8}=\frac{1}{\sqrt{3}}(3 F-D)=0.397 \pm 0.020
$$

where $F$ and $D$ are defined above (equation 16). This value is obtained by taking $F+D$ from equation (47) and $F / D$ from [52].

There is no theoretical prediction for $a_{0}$. However, using the measured value of $\Gamma_{p}$ from (45) and the values of $a_{3}$ and $a_{8}$ from (47) and (48), equation (46) gives

$$
a_{0}=0.098 \pm 0.076 \pm 0.113
$$

$S U(3)_{F}$ symmetry is not exact and this introduces an uncertainty in the value of $a_{8}$. For example, another measurement of F/D [54] would give $a_{8}=0.345 \pm .012$. However, it can be seen from equation (46) that the value of $a_{0}$ is not very sensitive to the value of $a_{8}$, and any uncertainty from the possible magnitude of $S U(3)_{F}$ symmetry breaking effects is much smaller than the experimental errors.

In the naïve parton model the $a_{j}$ are given by

$$
\begin{aligned}
& a_{0}=\sqrt{\frac{2}{3}}\{\Delta u+\Delta \bar{u}+\Delta d+\Delta \bar{d}+\Delta s+\Delta \bar{s}\} \\
& a_{3}=\{\Delta u+\Delta \bar{u}-\Delta d-\Delta \bar{d}\} \\
& a_{8}=\frac{1}{\sqrt{3}}\{\Delta u+\Delta \bar{u}+\Delta d+\Delta \bar{d}-2(\Delta s+\Delta \bar{s})\}
\end{aligned}
$$

where $\Delta q=\int_{0}^{1}\left(q^{+}(x)-q^{-}(x)\right) d x$. The Ellis-Jaffe sum rule (equation 17) was derived from equations (46) and (50) by assuming that $\Delta s=\Delta \bar{s}=0$, so that $a_{0}=\sqrt{2} a_{8}$. 
Assuming the validity of equations (50), we can now solve for the mean $z$ component of spin carried by each of the quark flavours in a proton with $S_{z}=+\frac{1}{2}$. This gives

$$
\begin{gathered}
<S_{z}>_{u}=\frac{1}{2}(\Delta u+\Delta \bar{u})=0.391 \pm 0.016 \pm 0.023 \\
<S_{z}>_{d}=\frac{1}{2}(\Delta d+\Delta \bar{d})=-0.236 \pm 0.016 \pm 0.023 \\
<S_{z}>_{s}=\frac{1}{2}(\Delta s+\Delta \bar{s})=-0.095 \pm 0.016 \pm 0.023 \\
\text { and }<S_{z}>_{\text {quarks }}=\frac{1}{2}(\Delta u+\Delta \bar{u}+\Delta d+\Delta \bar{d}+\Delta s+\Delta \bar{s}) \\
=\frac{1}{2} \sqrt{\frac{3}{2}} a_{0}=+0.060 \pm 0.047 \pm 0.069 .
\end{gathered}
$$

Thus, as expected, the $u$ quarks are predominantly polarised parallel to the proton and the $d$ quarks antiparallel. However, in this model, the strange quarks are also polarised antiparallel to the proton and only (12 $\pm 9 \pm 14) \%$ of the spin of the proton arises from the spin of its constituent quarks. Assuming, further, that the light quark sea is symmetric between $u$ and $d$ quarks and polarised to the same extent as that for strange quarks, together with the assumption that there are twice as many $u$ or $d$ quarks in the sea as strange quarks [1], it follows that

$$
\begin{aligned}
<S_{z}>_{\text {valence }} & =\frac{1}{2}(\Delta u+\Delta d-4 \Delta s) \\
& =+0.535 \pm 0.032 \pm 0.046 \\
<S_{z}>_{\text {sea }} & =5 \Delta s=-0.475 \pm 0.080 \pm 0.115
\end{aligned}
$$

Hence, with these assumptions, we see that the spin of the valence quarks completely accounts for the spin of the proton, but that it is almost exactly cancelled out by an equal and opposite contribution from the sea quarks.

These are surprising results in view of the success of the static quark model in explaining such phenomena as the ratio of the neutron to proton magnetic moments. In this it is assumed that the spin of the nucleon is due entirely to the spin of its valence quarks, without a contribution from sea quarks.

The validity of the result (equation 45) was initially questioned [55] on the grounds that $g_{1}^{p}(x)$ could diverge as $x$ tends to zero, giving a large contribution to the integral from the unmeasured region $(x \leq 0.01)$. It was argued that $g_{1}^{p}(x)$ could vary like $1 / x \ln ^{2} x$ at small $x$ as was once suggested on the basis of the Pomeron-Pomeron cut [56]. Such behaviour is no longer favoured on theoretical grounds $[48,57,58,59]$ and, although it cannot definitely be excluded, there is no divergent tendency in the data (fig. 12). In addition, the integral converges well as $x$ tends to zero (fig. 13). 
It has also been suggested [59] that there could be significant higher twist effects which would reduce the value of $\Gamma_{p}$ in the $Q^{2}$ range of this experiment and partially explain the low measured value. To check this idea the data have been split into two $Q^{2}$ ranges, above and below $8 \mathrm{GeV}^{2}$, and $\Gamma_{p}$ determined in each range. In the lower $Q^{2}$ range, combining the EMC and SLAC data and using the averaged $F_{2}$ as described above (see section 9.1), the result is $\Gamma_{p}\left(Q^{2}\right)=0.130 \pm .015 \pm .018$ at a mean $Q^{2}=4.8 \mathrm{GeV}^{2}$. In the upper $Q^{2}$ range, using the EMC data alone and the same averaged $F_{2}$ as above, the result is $\Gamma_{p}\left(Q^{2}\right)=0.114 \pm .021 \pm .019$ at a mean $Q^{2}$ of $17.2 \mathrm{GeV}^{2}$. It can thus be seen that there is no significant $Q^{2}$ dependence of $\Gamma_{p}$ in the range covered by the data. This was also to be expected from the very weak $Q^{2}$ dependence of $A_{1}^{p}$ at fixed $x$ (see fig. 7). Hence we conclude that the higher twist effects in the $Q^{2}$ range of this experiment are probably not large.

An explanation of the effect has been given $[57,60]$ using the Skyrme model of the nucleon in the chiral limit of massless quarks and in the leading order of the $\frac{1}{N_{c}}$ expansion. In this model it is shown that none of the spin of the proton is carried by the quark spins. Even when chiral symmetry and $\mathrm{SU}(3)$ are broken, the contribution of the quark spins to the proton spin is still small. If the chiral Lagrangian is adjusted so that the gluons carry $50 \%$ of the momentum of the proton, then most of the orbital angular momentum $L_{z}$ is carried by the quarks and this accounts for the proton spin [48].

An alternative explanation of the effect comes from the triangle anomaly of QCD. Recently it has been pointed out $[61,62,63,64,65]$ that the picture represented by equations (50) is too naive, since QCD radiative corrections arising from the Adler, Bell, Jackiw triangle anomaly $[66,67]$ have been neglected. When this is taken into account, each of the terms $\Delta q+\Delta \bar{q}$ in equations (50) is replaced by $\Delta q+\Delta \bar{q}-\left(\alpha_{s} / 2 \pi\right) \Delta g$, where $\Delta g=$ $\int_{0}^{1}\left(g^{+}(x)-g^{-}(x)\right) d x$ is the mean $z$-component of spin of the gluons in a proton with $S_{z}=+\frac{1}{2}$. Hence $a_{0}$ should be written

$$
a_{0}=2 \sqrt{\frac{2}{3}}\left(<S_{z}>_{\text {quarks }}-\frac{3 \alpha_{s}\left(Q^{2}\right)}{4 \pi}<S_{z}>_{\text {gluons }}\right) .
$$

If we now assume that $\left\langle S_{z}\right\rangle_{\text {quarks }} \simeq 0.35$ as expected from the Ellis-Jaffe sum rule, then it can be seen from equations (49) and (53) that $\Delta g$ is rather large $\left(\Delta g \sim 5\right.$, at $Q^{2}=$ $\left.10.7 \mathrm{GeV}^{2}\right)$. Such an effect is predicted from dynamically generated spin dependent parton distributions[68]. Since $\alpha_{8} \Delta g$ is approximately constant, independent of $Q^{2}$, then at low $Q^{2}$, where $\alpha_{s}$ is large, $\Delta g$ would be small and the static quark picture would prevail. Furthermore, the triangle anomaly can be used to explain why $g_{A}=1.25$ and not $5 / 3$ as expected from $\mathrm{SU}(6)$ symmetry [69]. Since each of the numerical results on the right hand side of equations (51) now refers to the combination $\frac{1}{2}\left(\Delta q+\Delta \bar{q}-\left(\alpha_{s} / 2 \pi\right) \Delta g\right)$, with the above value of $\Delta g$ at $Q^{2} \simeq 10 \mathrm{GeV}^{2},<S_{z}>_{s}=\frac{1}{2}(\Delta s+\Delta \bar{s}) \simeq 0$.

Several other possible explanations for the result have been offered including the sugges- 
tion that it is evidence against QCD [70]. To separate these different possibilities will require further measurements, covering as wide a $Q^{2}$ range as possible. Furthermore, it is of crucial importance to measure the asymmetries from a target containing polarised neutrons in order to test the highly fundamental Bjorken sum rule.

\section{ACKNOWLEDGEMENTS}

We wish to thank all those people in our home institutions who have contributed to the construction and maintenance of the apparatus. We especially thank G J Alner, Y Camp, E Cumiskey, R Gray, C Rigoni, J Thadome and E Watson who did so much to make this experiment possible. We also thank the polarised target groups at CERN, Liverpool and the Rutherford Appleton Laboratory for their sterling efforts in constructing the largest polarised target to be built to date. In addition we thank the CERN directorate for its support, the SPS division for the provision of an excellent source of polarised muons and DD division for its support and help. We dedicate this paper to the memory of the late Roger Gray.

\section{REFERENCES}

[1] For recent reviews see:

J Drees and H E Montgomery; Ann. Rev. Nucl. Part. Sci. $\underline{33}$ (1983) 383.

T Sloan, G Smadja and R Voss; Phys. Rep. 162 (1988) 45.

M Diemoz, F Ferroni and E Longo; Phys. Rep. 130 (1986) 295.

[2] SLAC-Yale, E80, M J Alguard et al, Phys. Rev. Lett. $\underline{37}$ (1976) 1261 and $\underline{41}$ (1978)70.

[3] SLAC-Yale E130, G Baum et al, Phys. Rev. Lett. $\underline{51}$ (1983) 1135.

[4] SLAC-Yale, G Baum et al, Phys. Rev. Lett. $4 \underline{5}$ (1980) 2000.

[5] J Ellis and R L Jaffe, Phys. Rev. D9 (1974) 1444 and D10 (1974) 1669.

[6] T Jones, Ph.D Thesis, University of Liverpool (1987).

D Salmon, Ph.D Thesis, University of Sheffield (1989).

R Piegaia, Ph.D Thesis, Yale University (1988).

V Papavassiliou, Ph.D Thesis, Yale University (1988).

M Matthews, Ph.D Thesis, University of Liverpool (1989).

[7] EMC, J Ashman et al, Phys. Lett. 206B (1988) 364.

[8] V W Hughes et al, Phys. Lett. 212B (1988) 511.

[9] For a review of the notation and previous work see V W Hughes and J Kuti, Ann. Rev. of Nuc. and Part. Science $\underline{33}$ (1983) 611 and E Gabathuler, Proc. 6th Int. Symposium on High Energy Spin Physics, Marseille, ed. J Soffer (1984).

[10] J D Bjorken, Phys. Rev. 179 (1969) 1547. 
[11] E Leader and E Predazzi,An Introduction to Gauge Theories and The New Physics (Cambridge University Press, 1982).

[12] M G Doncel and E de Rafael, Nuovo Cimento 4A (1971) 363.

[13] M Anselmino and E Leader, Z. Phys. C $\underline{41}$ (1988) 239.

[14] F E Close, An Introduction to Quarks and Partons (Academic Press, 1979).

[15] H Cheng and E Fischbach, Phys. Rev. D19 (1979) 860.

[16] D J E Callaway and S D Ellis, Phys. Rev. D29 (1984) 567.

[17] R Carlitz and J Kaur, Phys. Rev. Lett. $\underline{38}$ (1977) 673 and $\underline{38}$ (1977) 1102.

J Kaur, Nucl. Phys. B128 (1977) 219.

[18] J Schwinger, Nucl. Phys. B123 (1977) 223.

[19] G Farrar and J D Jackson, Phys. Rev. Lett. $\underline{35}$ (1975) 1416.

[20] J D Bjorken, Phys. Rev. 148 (1966) 1467;

J D Bjorken, Phys. Rev. D1 (1970) 1376.

[21] J Kodaira et al, Phys. Rev. D20 (1979) 627;

J Kodaira et al, Nucl. Phys. B159 (1979) 99;

J Kodaira, Nucl. Phys. B165 (1980) 129.

[22] R P Feynman, Photon-Hadron Interactions (Benjamin, New York 1972).

[23] J J de Swart, Rev. Mod. Phys. $\underline{35}$ (1963) 916.

[24] EMC, O C Allkofer et al, Nucl. Inst. and Meth. $\underline{179}$ ( 1981) 445.

[25] S C Brown et al, Proc. 4th International Workshop on polarised target materials and techniques (Bonn 1984) ed. W Meyer.

[26] N H Cunliffe and R L Roberts, Proc. 7th Int. Conf. on Cryogenic Engineering, London, 1978 (IPC Science and Technology Press) 102.

[27] T O Niinikoski, Nucl. Inst. and Meth. $\underline{192}$ (1982) 151.

[28] G R Court and W G Heyes, Nucl. Inst. and Methods A243 (1986) 37.

[29] Ch. Iselin, HALO, A Computer Program to Calculate Muon Halo, CERN Yellow Report 74-17 (1974).

[30] D Bollini et al, Nuovo Cimento $\underline{63 \mathrm{~A}}$ (1981) 441.

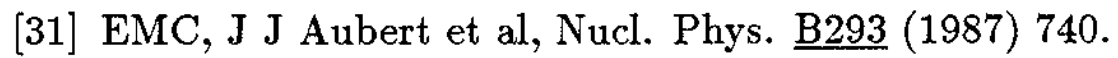

[32] Preliminary BCDMS results can be found in T Sloan et al. ref. 1. 
[33] EMC, J J Aubert et al, Phys. Lett. 123B (1983) 275.

[34] EMC, J Ashman et al, Phys. Lett. 202B (1988) 603 and M Duren, Ph.D Thesis, University of Aachen (1987).

[35] M Glück and E Reya, Nucl. Phys. B145 (1978) 24.

[36] L W Mo and Y S Tsai, Rev. Mod. Phys. 41 (1965) 205;

Y S Tsai, SLAC Report SLAC-PUB-848 (1971), unpublished.

[37] T V Kukhto and N M Shumeiko, Yad. Fiz. $\underline{36}$ (1982) 707.

[38] SLAC-Yale, E-80, M J Alguard et al, Phys. Rev. Lett. 37 (1976) 1258.

[39] A Schäfer, Phys. Lett. 208B (1988) 175.

[40] A Giannelli et al, Phys. Lett. 150B (1985) 214.

[41] O Darrigol and F Hayot, Nucl. Phys. B141 (1978) 391.

[42] EMC, J J Aubert et al, Phys. Letts. 160B (1985) 417.

[43] R L Heimann, Nucl. Phys. B121 (1977) 145 and J. Phys. G, Nucl. Phys. $\underline{4}$ (1978) 173.

[44] M Diemoz, F Ferroni, E Longo and G Miriiuelli, Z. Phys. Cㅉ9 (1988) 21.

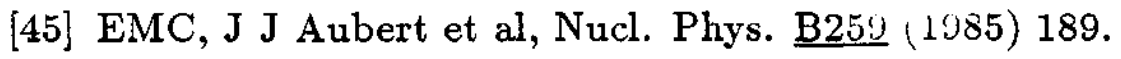

[46] B L Ioffe, V A Khoze, L N Lipatov, Hard Processes (North-Holland, Amsterdam, 1984) page 61 .

[47] EMC (NA28), to be published.

[48] J Ellis and M Karliner, Phys. Lett. 213B (1988) 73.

[49] R Voss, 1987 International Symposium on Lepton and Photon Interactions at High Energies, Hamburg.

[50] EMC, J J Aubert et al, Nucl. Phys. B272 (1986) 158.

[51] BCDMS, A Ouraou, Ph.D Thesis, Saclay (1988) and A C Benvenuti et al, CERN preprint, CERN-EP/89-06.

[52] M Bourquin et al, Z. Phys. C21 (1983) 27.

[53] See for example, M Glück and E Reya, Z. Phys. C39 (1988) 569.

[54] S Y Hsueh et al, Phys. Rev. D38 (1988) 2056.

[55] F Close and R G Roberts, Phys. Rev. Letts. $\underline{60}$ (1988) 1471. 
[56] L Gàffi, P Gnädig, J Kuti, F Niedermayer and A Patkòs, Acta. Phys. Acad. Sci. Hung. $\underline{31}$ (1972) 85 .

[57] S J Brodsky, J Ellis, M Karliner, Phys. Letts. B206 (1988) 309.

[58] G P Ramsey, J W Qiu, D Richards, D Sivers, Phys. Rev. D39 (1989) 361.

[59] M Anselmino, B L Ioffe, E Leader, preprint NSF-ITP-88-94.

[60] Z Ryzak, Phys. Letts. B217 (1989) 325.

[61] R L Jaffe, Phys. Lett. B193 (1987) 101.

[62] A V Efremov and O V Teryaev, Dubna preprint E2-88-287.

[63] G Altarelli and G G Ross, Phys. Lett. 212B (1988) 391.

[64] R D Carlitz, J C Collins, A H Mueller, Phys. Letts. 214B (1988) 229.

[65] E Leader, Birkbeck College Preprint, (1988).

[66] S L Adler, Phys. Rev. 177 (1969) 2426.

[67] J S Bell and R Jackiw, Nuovo Cim. $\underline{60 A}$ (1969) 47.

[68] M Glück and E Reya, Dortmund Preprint Do. Th. 88/22.

[69] H Fritzsch Preprint MPI-PAE/PTH-18/89 and Munich Preprint 88-0570 (1988).

[70] G Preparata and J Soffer, Phys. Rev. Letts. $\underline{61}$ (1988) 1167.

\begin{tabular}{|l|l|}
\hline \multicolumn{2}{|c|}{ Table 1: Definition of the kinematic variables used } \\
\hline $\mathrm{m}$ & Lepton rest mass \\
$\mathrm{M}$ & Proton rest mass \\
$\mathrm{s}=\frac{1}{m}(\mathrm{k}, 0,0, \mathrm{E})$ & Lepton-spin four-vector \\
$\mathrm{S}=(0, \vec{S})$ & Proton-spin four-vector \\
$\mathrm{k}=(\mathrm{E}, \vec{k})$ & Four-momentum of incident lepton \\
$\mathrm{k}^{\prime}=\left(\mathrm{E}^{\prime}, \vec{k}^{\prime}\right)$ & Four-momentum of scattered lepton \\
$\mathrm{P}=(\mathrm{M}, \overrightarrow{0})$ & Four-momentum of target proton \\
$\mathrm{q}=\mathrm{k}-\mathrm{k}^{\prime}=(\nu, \vec{q})$ & Four momentum transfer \\
$\mathrm{Q}^{2}=-\mathrm{q}^{2} \simeq 4 \mathrm{EE}^{\prime} \sin ^{2}(\theta / 2)$ & (Invariant mass) of virtual photon \\
$\nu=\mathrm{P} \cdot \mathrm{q} / \mathrm{M}=\mathrm{E}-\mathrm{E}^{\prime}$ & Energy of the virtual photon in the laboratory \\
$\theta$ & Scattering angle in the laboratory \\
$\mathrm{x}=\mathrm{Q}^{2} / 2 \mathrm{M} \nu$ & Bjorken scaling variable \\
$\mathrm{y}=\nu / \mathrm{E}$ & Bjorken scaling variable \\
\hline
\end{tabular}




\begin{tabular}{|c|c|}
\hline \multicolumn{2}{|c|}{ Table 2: Beam-polarisation values calculated by Monte Carlo } \\
\hline Energy: $\mathrm{E}_{\pi} / \mathrm{E}_{\mu}(\mathrm{GeV})$ & Polarisation \\
\hline $110 / 100$ & $0.77 \pm 0.06$ \\
$130 / 120$ & $0.79 \pm 0.06$ \\
$210 / 200$ & $0.82 \pm 0.06$ \\
\hline
\end{tabular}

Table 3: Kinematic cuts applied to the data for the three beam energies

\begin{tabular}{|c|c|c|c|c|c|}
\hline $\begin{array}{c}\mathrm{E}_{\mu} \\
(\mathrm{GeV})\end{array}$ & $\begin{array}{c}\mathrm{Q}^{2} \min \\
\left(\mathrm{GeV}^{2} / \mathrm{c}^{2}\right)\end{array}$ & $\begin{array}{c}\nu_{\min } \\
(\mathrm{GeV})\end{array}$ & $\begin{array}{c}\mathrm{E}_{\mu}^{\prime}, \min \\
(\mathrm{GeV})\end{array}$ & $\mathrm{y}_{\max }$ & $\theta_{\min }$ \\
\hline 100 & 1.5 & 10 & 18 & 0.85 & $1^{0}$ \\
120 & 2.0 & 10 & 20 & 0.85 & $1^{0}$ \\
200 & 3.0 & 20 & 30 & 0.85 & $1^{0}$ \\
\hline
\end{tabular}

\begin{tabular}{|l|c|c|c|c|}
\hline \multicolumn{5}{|c|}{ Table 4: Data used to measure the asymmetry. } \\
\hline $\begin{array}{l}\text { Run } \\
\text { period, year) }\end{array}$ & $\begin{array}{c}\text { Energy } \\
(\mathrm{GeV})\end{array}$ & $\begin{array}{c}\text { Initial target } \\
\text { orientations }\end{array}$ & $\begin{array}{c}\text { Mean target } \\
\text { polarisation, } P_{T} \\
(\%)\end{array}$ & $\begin{array}{c}\text { No. of Events } \\
\text { after cuts }\end{array}$ \\
\hline 2B84 & 200 & $-/+$ & 77.3 & $114.6 \mathrm{~K}$ \\
2C84 I & 200 & $-/+$ & 78.5 & $62.5 \mathrm{~K}$ \\
2C84 II & 200 & $+/-$ & 75.5 & $68.7 \mathrm{~K}$ \\
3A84 & 120 & $-/+$ & 74.4 & $236.3 \mathrm{~K}$ \\
3B84 & 200 & $+/-$ & 78.7 & $115.8 \mathrm{~K}$ \\
3C84 I & 200 & $-/+$ & 79.0 & $44.1 \mathrm{~K}$ \\
3C84 II & 100 & $+/-$ & 80.7 & $202.1 \mathrm{~K}$ \\
2A85 & 200 & $-/+$ & 80.5 & $41.5 \mathrm{~K}$ \\
2B85 I & 120 & $-/+$ & 72.7 & $180.5 \mathrm{~K}$ \\
2B85 II & 200 & $+/-$ & 71.7 & $58.5 \mathrm{~K}$ \\
2C85 & 200 & $-/+$ & 78.4 & $97.5 \mathrm{~K}$ \\
\hline
\end{tabular}




\begin{tabular}{|c|c|c|c|c|c|c|c|c|c|c|c|c|}
\hline & 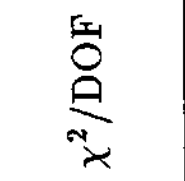 & $\stackrel{9}{\stackrel{9}{N}}$ & 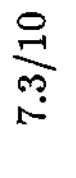 & $\underset{\substack{\text { No } \\
20}}{\stackrel{0}{1}}$ & 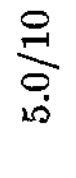 & 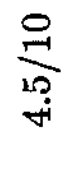 & 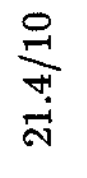 & 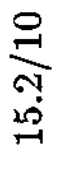 & 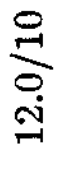 & $\underset{\substack{\stackrel{0}{1} \\
\stackrel{\infty}{\infty}}}{ }$ & $\underset{-\dot{\theta}}{\stackrel{O}{-}}$ & \\
\hline & 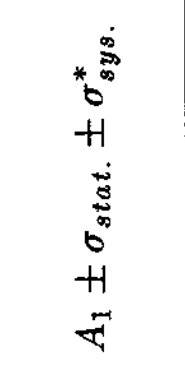 & 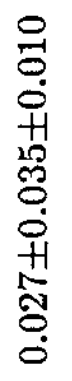 & 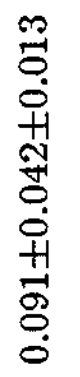 & 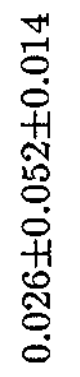 & 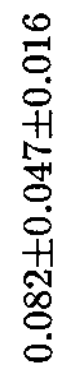 & 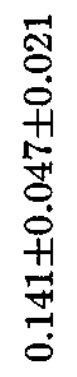 & 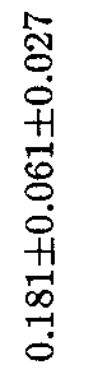 & 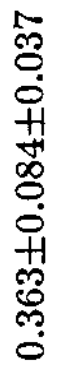 & $\begin{array}{l}\vec{Z} \\
0 \\
0 \\
+1 \\
00 \\
0 \\
0 \\
0 \\
+1 \\
00 \\
0 \\
0 \\
0\end{array}$ & 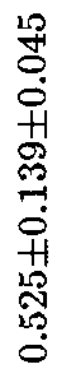 & 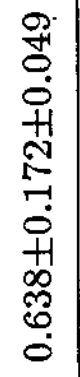 & 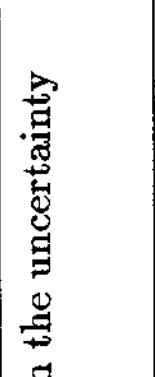 \\
\hline 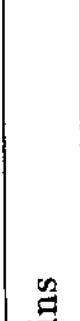 & 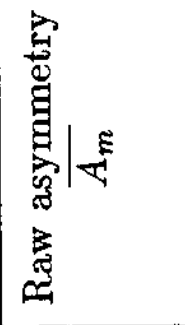 & $\begin{array}{l}\stackrel{0}{0} \\
\stackrel{8}{0} \\
\stackrel{0}{+} \\
+ \\
\stackrel{0}{8} \\
\stackrel{0}{0}\end{array}$ & 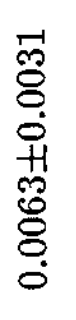 & 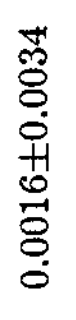 & $\begin{array}{l}\stackrel{5}{1} \\
8 \\
0 \\
0 \\
+1 \\
0 \\
0 \\
0 \\
0 \\
0\end{array}$ & 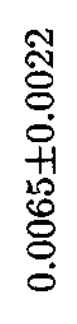 & $\begin{array}{l}\stackrel{2}{1} \\
8 \\
0 \\
\dot{0} \\
+1 \\
0 \\
0 \\
0 \\
0\end{array}$ & 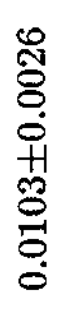 & 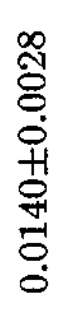 & 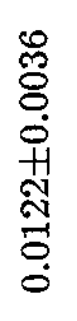 & 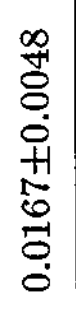 & 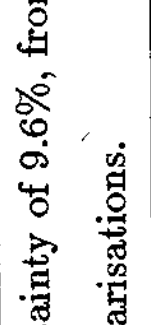 \\
\hline 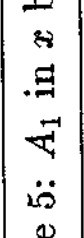 & 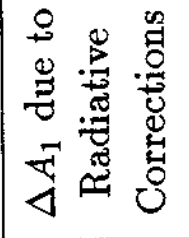 & $\stackrel{22}{8}$ & $\begin{array}{l}20 \\
8 \\
0 \\
0\end{array}$ & $\stackrel{20}{8}$ & $\begin{array}{l}20 \\
8 \\
0\end{array}$ & $\begin{array}{l}\stackrel{J}{8} \\
\stackrel{8}{0}\end{array}$ & 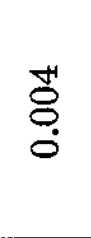 & $\stackrel{20}{8}$ & $\stackrel{5}{8}$ & $\stackrel{\overrightarrow{0}}{\stackrel{0}{0}}$ & $\stackrel{ }{\circ}$ & 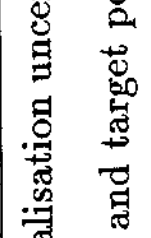 \\
\hline${ }_{E \rightarrow H}^{\circ}$ & $\stackrel{4}{\stackrel{5}{3}}$ & $\begin{array}{l}\vec{\infty} \\
\stackrel{1}{0}\end{array}$ & $\begin{array}{l}\infty \\
\stackrel{0}{0} \\
\end{array}$ & $\begin{array}{l}\overrightarrow{6} \\
\stackrel{1}{0}\end{array}$ & 望 & : & $\begin{array}{l}\text { 足 } \\
\stackrel{9}{-1} \\
0\end{array}$ & $\begin{array}{l}\mathscr{P} \\
\stackrel{0}{0} \\
0\end{array}$ & $\stackrel{\overrightarrow{1}}{\overrightarrow{0}}$ & $\begin{array}{l}\mathscr{\infty} \\
\stackrel{\infty}{0} \\
\stackrel{0}{0}\end{array}$ & $\stackrel{\mathscr{D}}{\stackrel{\mathscr{\sigma}}{\circ}}$ & 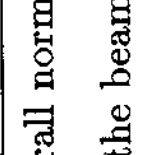 \\
\hline & 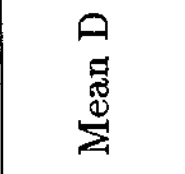 & $\stackrel{⿱ 1}{\stackrel{\infty}{0}}$ & $\begin{array}{l}\mathscr{8} \\
\stackrel{\leftrightarrow}{\circ}\end{array}$ & $\begin{array}{l}\text { ్ֶర } \\
\stackrel{0}{0} \\
0\end{array}$ & $\begin{array}{l}\text { No } \\
\text { @? } \\
0 \\
0\end{array}$ & 沾 & 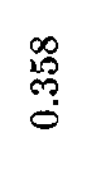 & 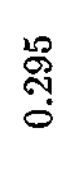 & 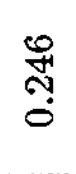 & $\begin{array}{l}\stackrel{\leftrightarrow}{A} \\
\stackrel{\text { N }}{\circ}\end{array}$ & 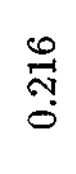 & $\frac{0}{0}$ \\
\hline & 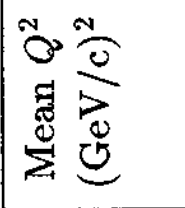 & קות & نه & $\stackrel{\circ}{0}$ & $\underset{\infty}{\infty}$ & $\stackrel{\leftrightarrow}{\circ}$ & $\stackrel{\stackrel{\leftrightarrow}{\sim}}{\stackrel{\sim}{\sim}}$ & 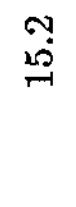 & $\stackrel{\circ}{\infty}$ & $\begin{array}{l}29 \\
\text { งิ่ }\end{array}$ & 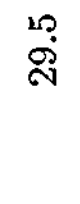 & 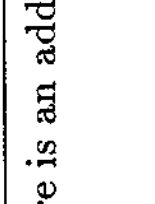 \\
\hline & 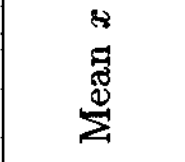 & $\stackrel{20}{0}$ & 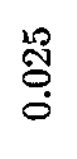 & $\begin{array}{l}20 \\
\stackrel{2}{0} \\
0\end{array}$ & $\begin{array}{l}\stackrel{\circ}{0} \\
\stackrel{2}{0} \\
0\end{array}$ & $\stackrel{\infty}{\stackrel{\infty}{0}}$ & $\stackrel{\vec{I}}{\stackrel{3}{0}}$ & $\stackrel{10}{0}$ & $\underset{\stackrel{\infty}{\sharp ! ~}}{\stackrel{\leftrightarrow}{0}}$ & 苞 & 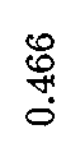 & E \\
\hline & 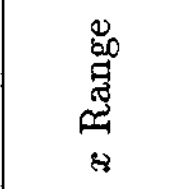 & $\begin{array}{l}\stackrel{9}{0} \\
0 \\
\dot{1} \\
\dot{1} \\
0 \\
0\end{array}$ & $\begin{array}{l}\mathscr{O} \\
\dot{0} \\
\dot{1} \\
\dot{0} \\
\dot{0}\end{array}$ & $\begin{array}{l}\ddot{0} \\
\stackrel{0}{0} \\
\stackrel{1}{0} \\
\stackrel{0}{0}\end{array}$ & $\begin{array}{l}0 \\
8 \\
0 \\
\dot{+} \\
0 \\
0\end{array}$ & $\begin{array}{l}0 \\
\stackrel{0}{0} \\
\dot{1} \\
\dot{0} \\
0 \\
0\end{array}$ & $\begin{array}{l}\stackrel{20}{0} \\
0 \\
0 \\
0 \\
0 \\
0\end{array}$ & 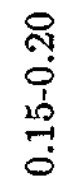 & 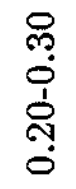 & $\begin{array}{l}\stackrel{9}{+} \\
\stackrel{0}{1} \\
\stackrel{1}{0} \\
\stackrel{0}{0}\end{array}$ & $\begin{array}{l}\text { 웅 } \\
0 \\
0 \\
0 \\
0 \\
0\end{array}$ & \\
\hline
\end{tabular}




\begin{tabular}{|c|c|c|c|c|c|c|}
\hline \multicolumn{7}{|c|}{ Table 6: Systematic errors for $A_{1}$} \\
\hline & $\mathrm{R}$ & $A_{2}$ & $\mathrm{~K}$ & $\mathrm{f}$ & Rad. corr. & Total $^{\dagger}$ \\
systematic
\end{tabular}

\begin{tabular}{|c|c|c|c|c|c|c|}
\hline $\mathbf{x}$ range & $<x>$ & $\begin{array}{c}<Q^{2}> \\
\left(\mathrm{GeV}^{2} / \mathrm{c}^{2}\right)\end{array}$ & $<D>$ & $<f>$ & $A^{+} \pm \sigma_{\text {stat }} \pm \sigma_{\text {syst }}^{\dagger}$ & $\chi^{2} /$ DoF \\
\hline $0.01-0.03$ & 0.020 & 4.2 & 0.713 & 0.177 & $0.122 \pm 0.057 \pm 0.028$ & $9.3 / 10$ \\
\hline $0.03-0.06$ & 0.044 & 7.6 & 0.582 & 0.164 & $-0.114 \pm 0.065 \pm 0.033$ & $17.6 / 10$ \\
\hline $0.06-0.15$ & 0.097 & 12.9 & 0.440 & 0.165 & $0.178 \pm 0.065 \pm 0.043$ & $7.6 / 10$ \\
\hline $0.15-0.30$ & 0.203 & 22.7 & 0.379 & 0.177 & $0.527 \pm 0.104 \pm 0.051$ & $7.5 / 10$ \\
\hline $0.30-0.70$ & 0.376 & 42.0 & 0.386 & 0.195 & $0.780 \pm 0.214 \pm 0.046$ & $16.1 / 10$ \\
\hline$x$ range & $<x>$ & $\begin{array}{c}<Q^{2}> \\
\left(\mathrm{GeV}^{2} / \mathrm{c}^{2}\right)\end{array}$ & $<D>$ & $<f>$ & $A^{-} \pm \sigma_{\text {stat }} \pm \sigma_{\text {syst }}^{\dagger}$ & $\chi^{2} / \mathrm{DoF}$ \\
\hline $0.01-0.03$ & 0.020 & 4.2 & 0.718 & 0.172 & $-0.021 \pm 0.064 \pm 0.024$ & $9.9 / 10$ \\
\hline $0.03-0.06$ & 0.044 & 7.7 & 0.584 & 0.157 & $0.012 \pm 0.074 \pm 0.033$ & $19.1 / 10$ \\
\hline $0.06-0.15$ & 0.098 & 12.9 & 0.444 & 0.156 & $0.002 \pm 0.077 \pm 0.043$ & $11.2 / 10$ \\
\hline $0.15-0.30$ & 0.203 & 22.4 & 0.374 & 0.164 & $0.269 \pm 0.131 \pm 0.051$ & $5.9 / 10$ \\
\hline $0.30-0.70$ & 0.371 & 41.1 & 0.379 & 0.178 & $0.562 \pm 0.283 \pm 0.047$ & $11.0 / 10$ \\
\hline
\end{tabular}




\begin{tabular}{|c|c|c|c|c|c|c|c|c|}
\hline \multirow{3}{*}{$x$} & \multicolumn{7}{|c|}{$\begin{array}{l}\text { Table 8: Final Results for the Spin-Dependent } \\
\text { Structure Function } g_{1}^{p}\end{array}$} & \multirow{3}{*}{$\begin{array}{c}\text { Total } \\
\text { Sys. } \\
\text { Error } \\
*\end{array}$} \\
\hline & \multirow{2}{*}{$g_{1}^{p}$} & \multirow{2}{*}{$\begin{array}{l}\text { Stat. } \\
\text { Error }\end{array}$} & \multicolumn{5}{|c|}{ Systematic error due to: } & \\
\hline & & & $\mathrm{R}$ & $A_{2}$ & $\mathrm{~K}$ & $f$ & $\begin{array}{l}\text { Rad. } \\
\text { Corr. }\end{array}$ & \\
\hline 0.015 & 0.279 & 0.361 & 0.014 & 0.046 & 0.093 & 0.018 & 0.009 & 0.106 \\
\hline 0.025 & 0.564 & 0.260 & 0.026 & 0.029 & 0.062 & 0.031 & 0.006 & 0.080 \\
\hline 0.035 & 0.115 & 0.230 & 0.004 & 0.021 & 0.052 & 0.004 & 0.004 & 0.057 \\
\hline 0.050 & 0.254 & 0.146 & 0.004 & 0.015 & 0.040 & 0.009 & 0.003 & 0.044 \\
\hline 0.078 & 0.280 & 0.093 & 0.005 & 0.009 & 0.032 & 0.012 & 0.002 & 0.036 \\
\hline 0.124 & 0.225 & 0.076 & 0.005 & 0.005 & 0.025 & 0.010 & 0.002 & 0.028 \\
\hline 0.175 & 0.311 & 0.072 & 0.003 & 0.003 & 0.021 & 0.014 & 0.003 & 0.026 \\
\hline 0.248 & 0.253 & 0.048 & 0.003 & 0.002 & 0.015 & 0.011 & 0.003 & 0.019 \\
\hline 0.344 & 0.167 & 0.044 & 0.002 & 0.001 & 0.010 & 0.008 & 0.002 & 0.013 \\
\hline 0.466 & 0.094 & 0.025 & 0.001 & 0.000 & 0.004 & 0.005 & 0.001 & 0.007 \\
\hline & & & & $4 \%$ & alisati & uncert & ty due & \\
\hline
\end{tabular}

\begin{tabular}{|ll|}
\hline $\begin{array}{c}\text { Table 9: The integral of } g_{1}^{p} \text { using different } \\
\text { measurements of the unpolarised structure function } F_{2}\end{array}$ \\
\hline & $\int_{0.01}^{0.7} g_{1}^{p} d x$ at $Q^{2}=10.7 \mathrm{GeV}^{2}$ \\
\hline Source of $F_{2}$ & $0.113 \pm .012$ \\
& $0.115 \pm .012$ \\
EMC Proton [45] & $0.123 \pm .013$ \\
EMC Iron [50] & $0.127 \pm .014$ \\
BCDMS [54] & 0.120 \\
Mean & 0.0068 \\
Standard Deviation & \\
\hline
\end{tabular}




\begin{tabular}{|lc|}
\hline \multicolumn{2}{|c|}{ Table 10: Systematic errors on the integral of $g_{1}$} \\
\hline Dilution Factor $f$ & \pm 0.0054 \\
Uncertainty in $R_{Q C D}$ & \pm 0.0007 \\
Radiative Corrections & \pm 0.0016 \\
Neglect of $A_{2}$ & \pm 0.0030 \\
Beam Polarisation & \pm 0.0092 \\
Target Polarisation & \pm 0.0074 \\
Uncertainty in $F_{2}$ & \pm 0.0071 \\
Acceptance Effects & \pm 0.0108 \\
Extrapolations into unmeasured region & \pm 0.0030 \\
& \\
Total Systematic Error & \pm 0.019 \\
\hline
\end{tabular}

\section{FIGURE CAPTIONS}

1. The EMC apparatus for the polarised-target experiment.

2. The polarised target.

3. Vertex distribution along the beam direction. The target edges and the applied cuts are shown.

4. Contributions from various sources to the radiative corrections; the curve labelled polarised proton correction is $B_{k}+T_{1}+T_{2}-1$, polarised nitrogen correction is $\frac{p_{T}^{N}}{p_{T}}\left(T_{3}+\right.$ $\left.T_{4}+T_{5}+T_{8}\right)$ and unpolarised correction is $f\left(T_{7}+T_{8}\right)$ (see text).

5. The correction factors for radiative effects; depolarisation factor, $D$; dilution factor, $f$; $<y>$ of the data as a function of $x$.

6. The asymmetry $A_{1}$ for the proton as a function of $x$ together with the results from previous experiments [2,3]. The curve is from the model of [17].

7. $A_{1}$ versus $Q^{2}$ in three $x$ bins.

8. Comparison of the asymmetries $A_{1}^{p}$ obtained from periods with the two possible initial polarised target configurations.

9. The measured value of $K-1$ obtained by comparing the asymmetries measured for events with muon tracks detected outside the chambers P4/5 and the total sample.

10. Comparison of the asymmetries $A_{1}^{p}$ as a function of $x$ for events with one or more detected hadrons with those from the total data sample. The smooth curve shows the prediction of the model of [17]. 
11. The semi-inclusive asymmetries $A^{+}\left(A^{-}\right)$for positive (negative) hadrons versus $x$.

12. The structure function $g_{1}^{p}(x)$ as a function of $x$. The dashed curve is the value deduced from the parameterisation (equation 35 ).

13. The convergence of the integral $\int_{x_{m}}^{1} g_{1}^{p} d x$ as a function of $x$, where $x_{m}$ is the value of $x$ at the low edge of each bin. 


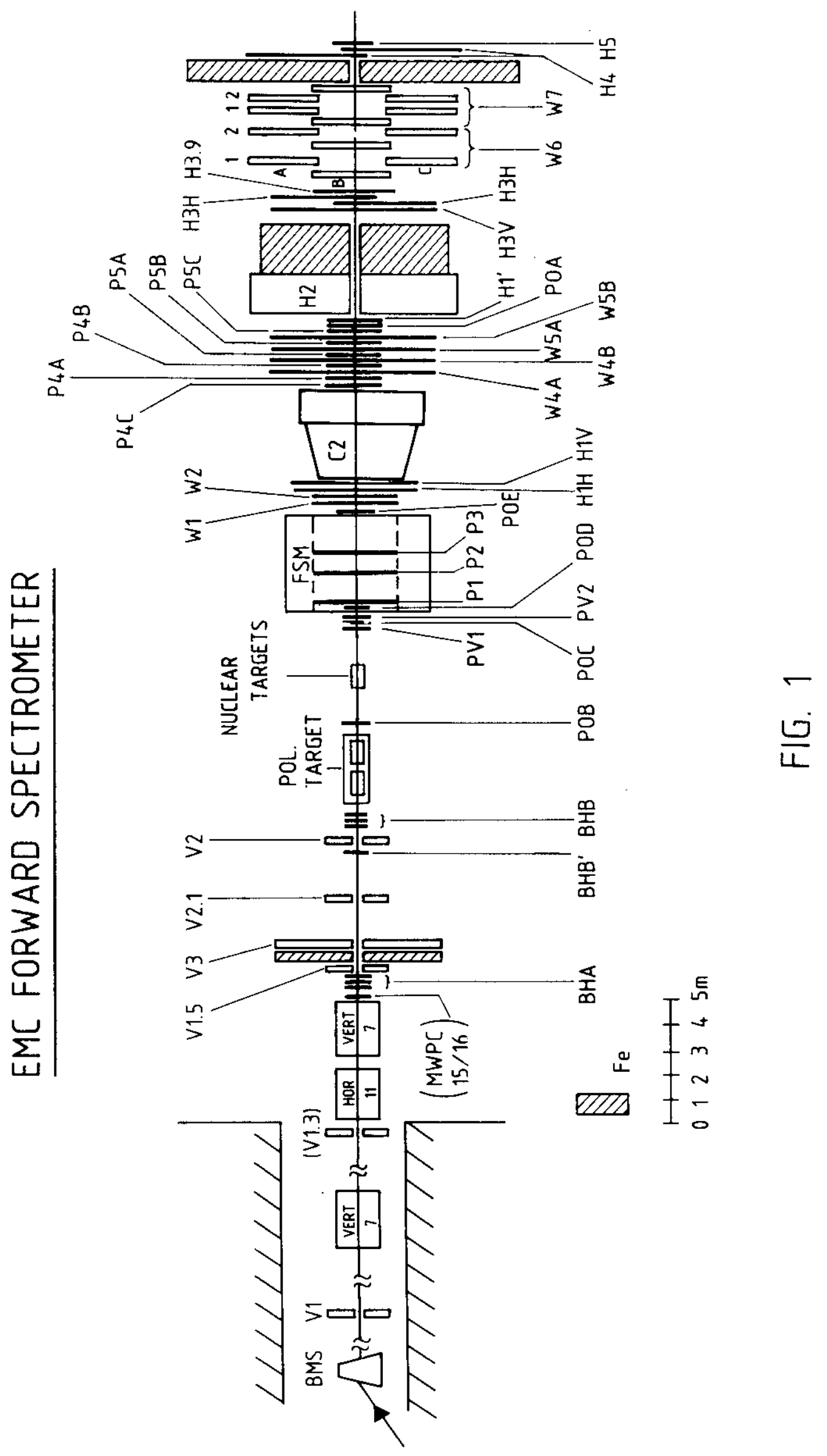




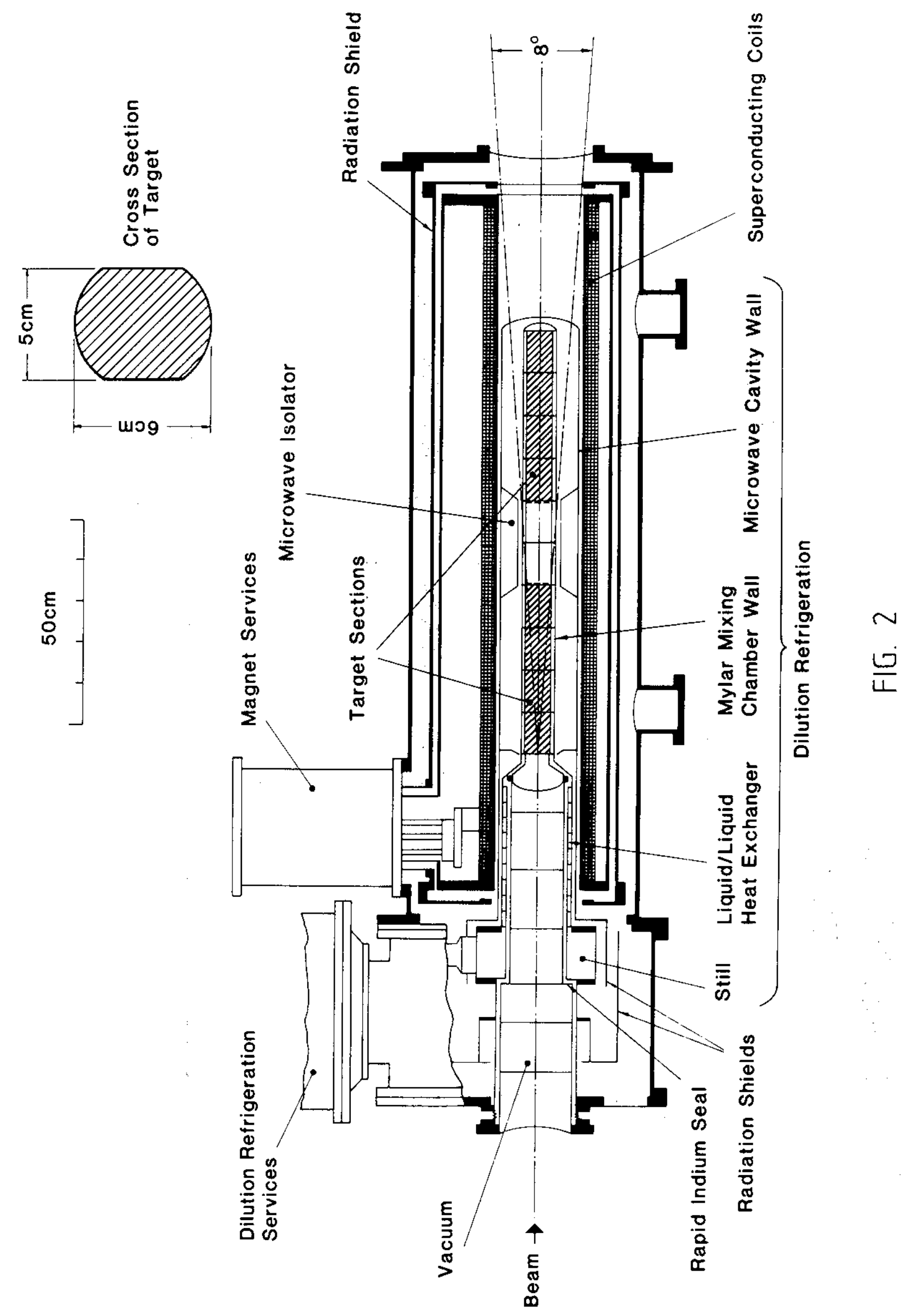




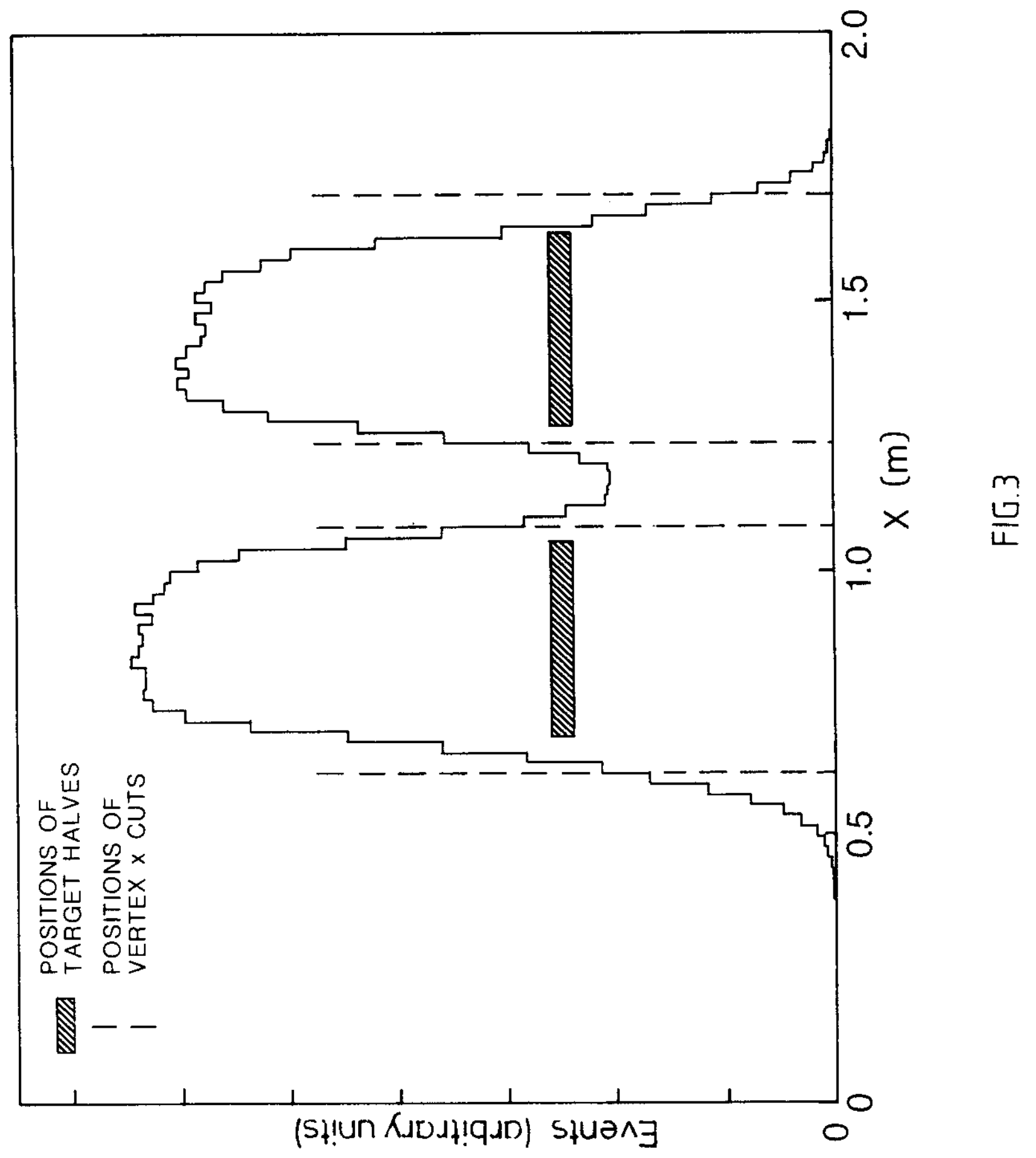




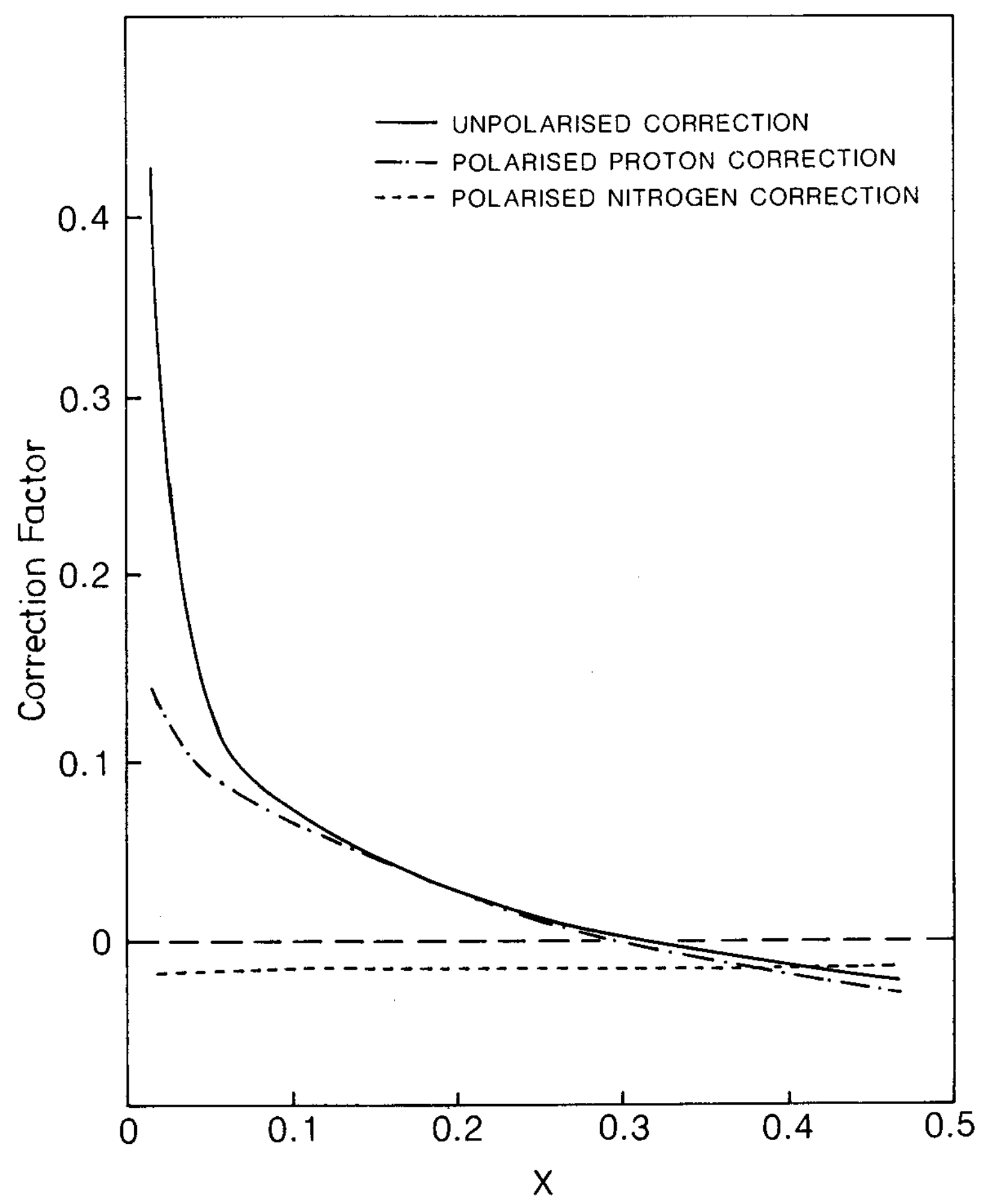

FIG. 4 


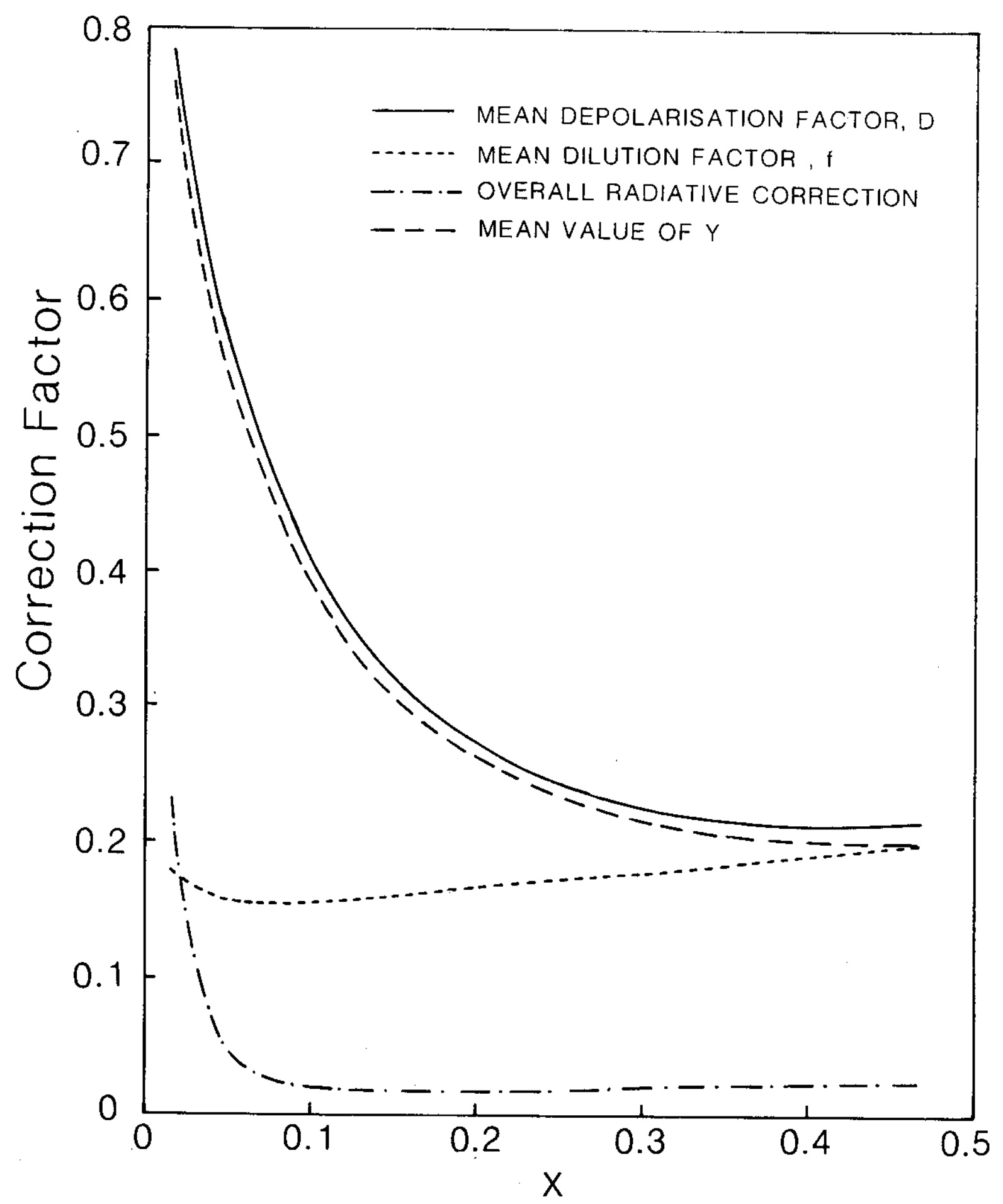

FIG. 5 


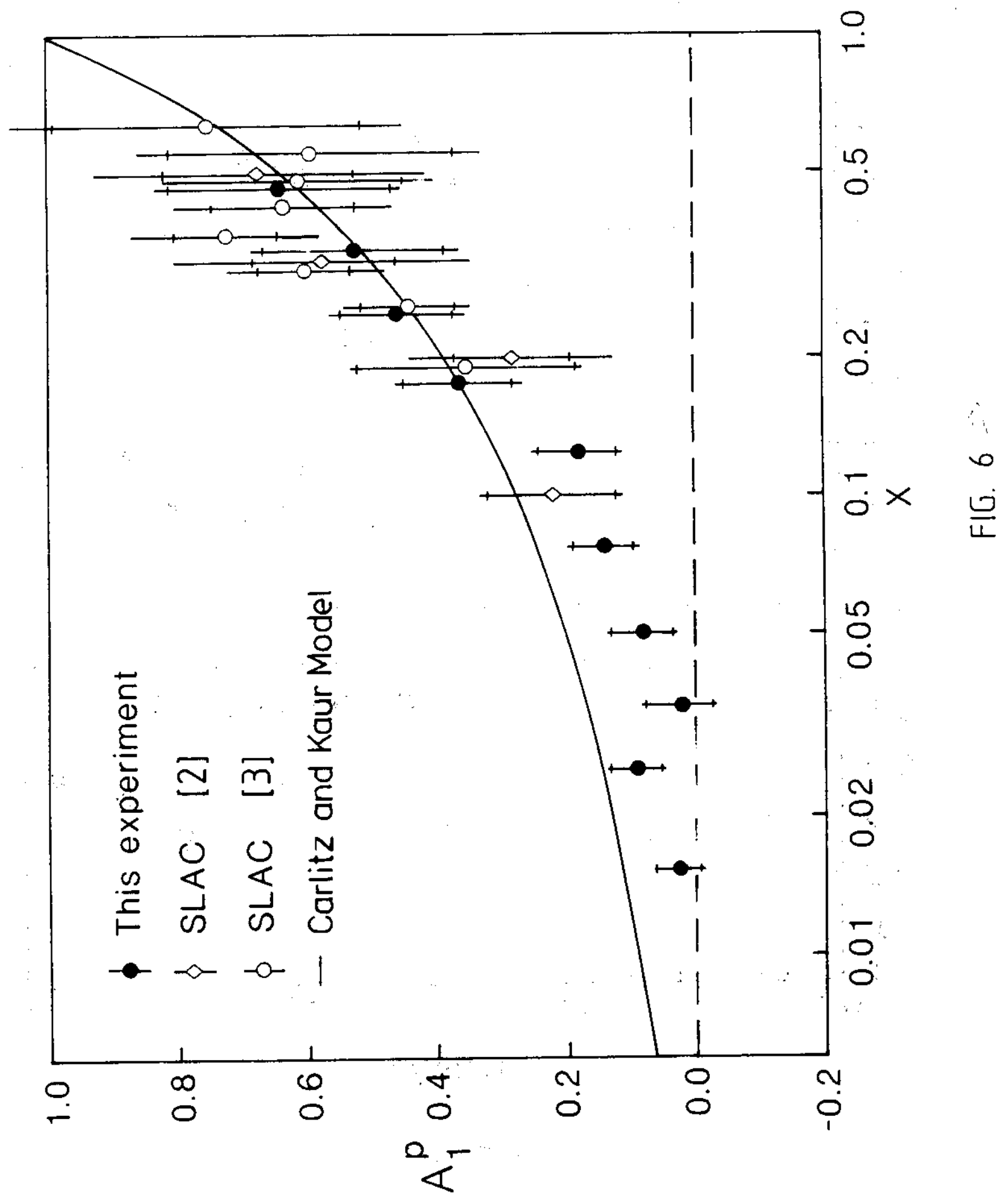




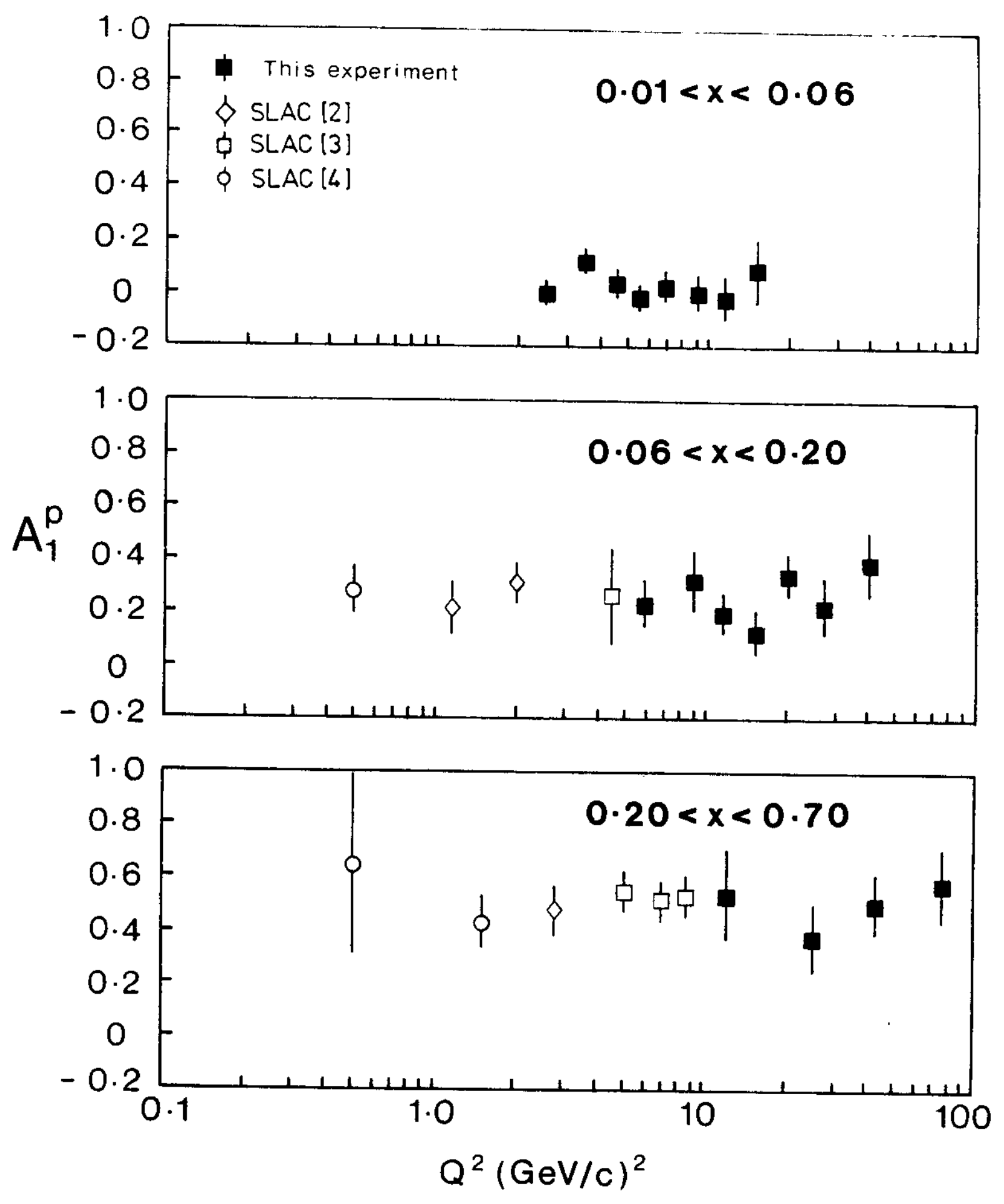

FIG. 7 


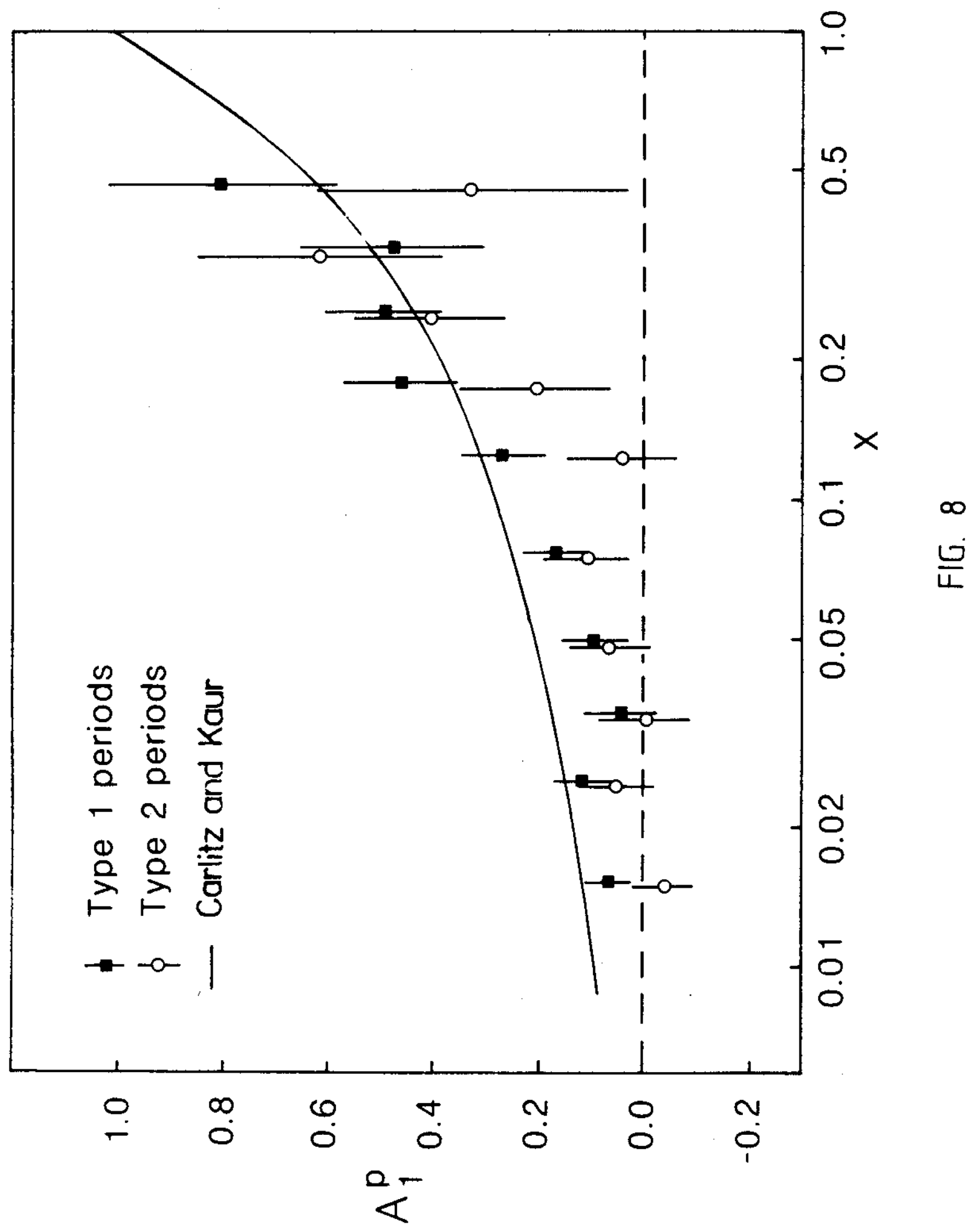




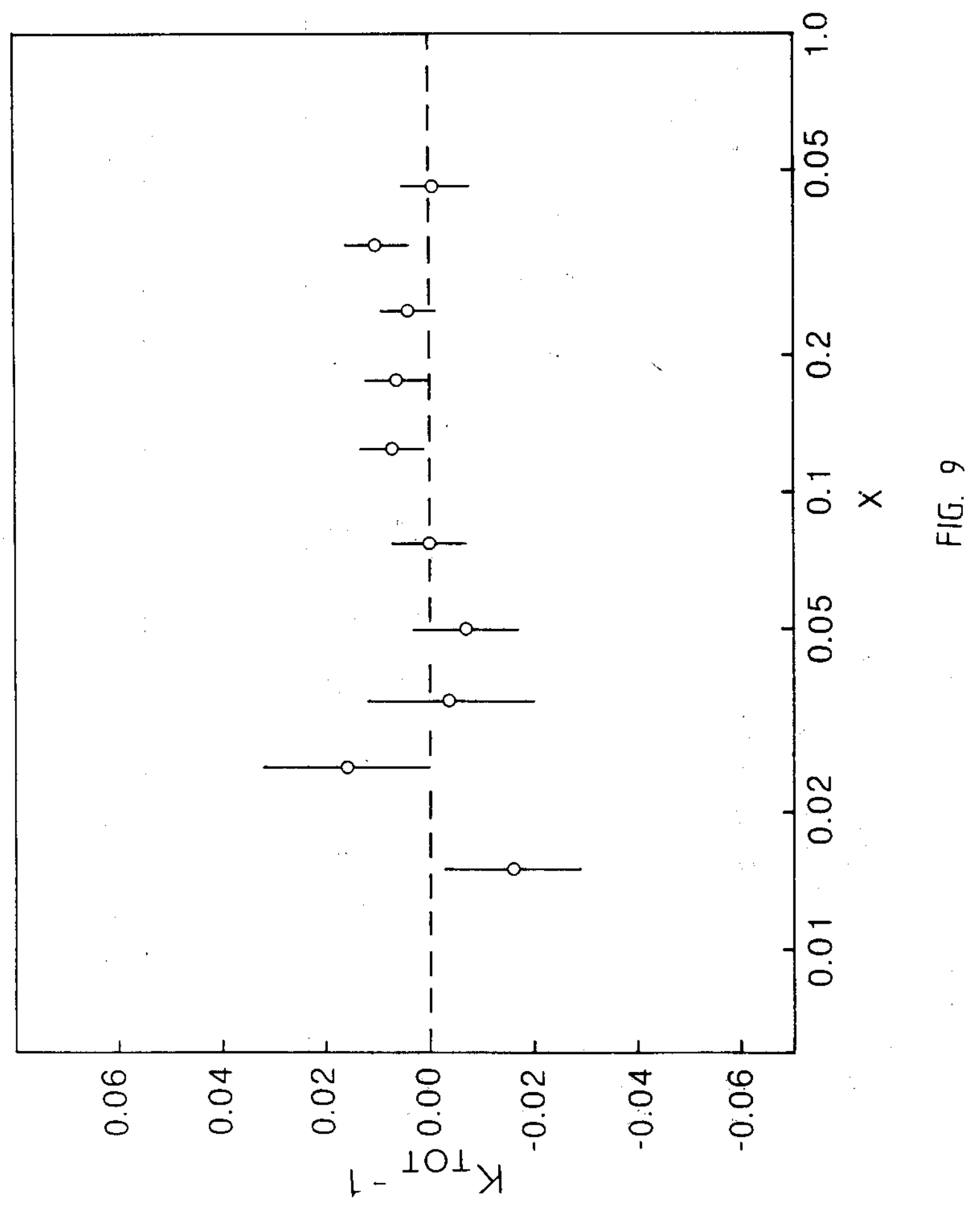




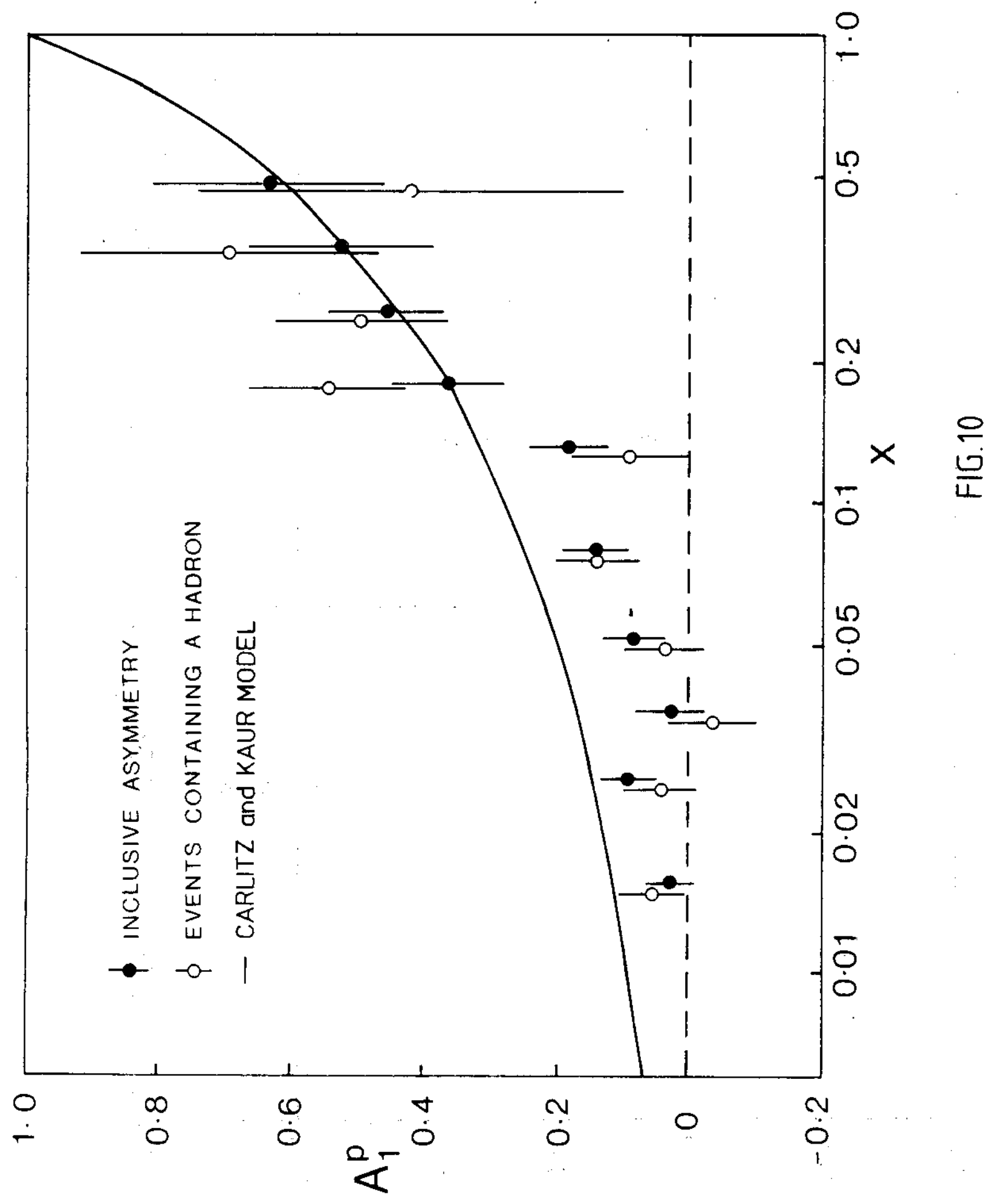




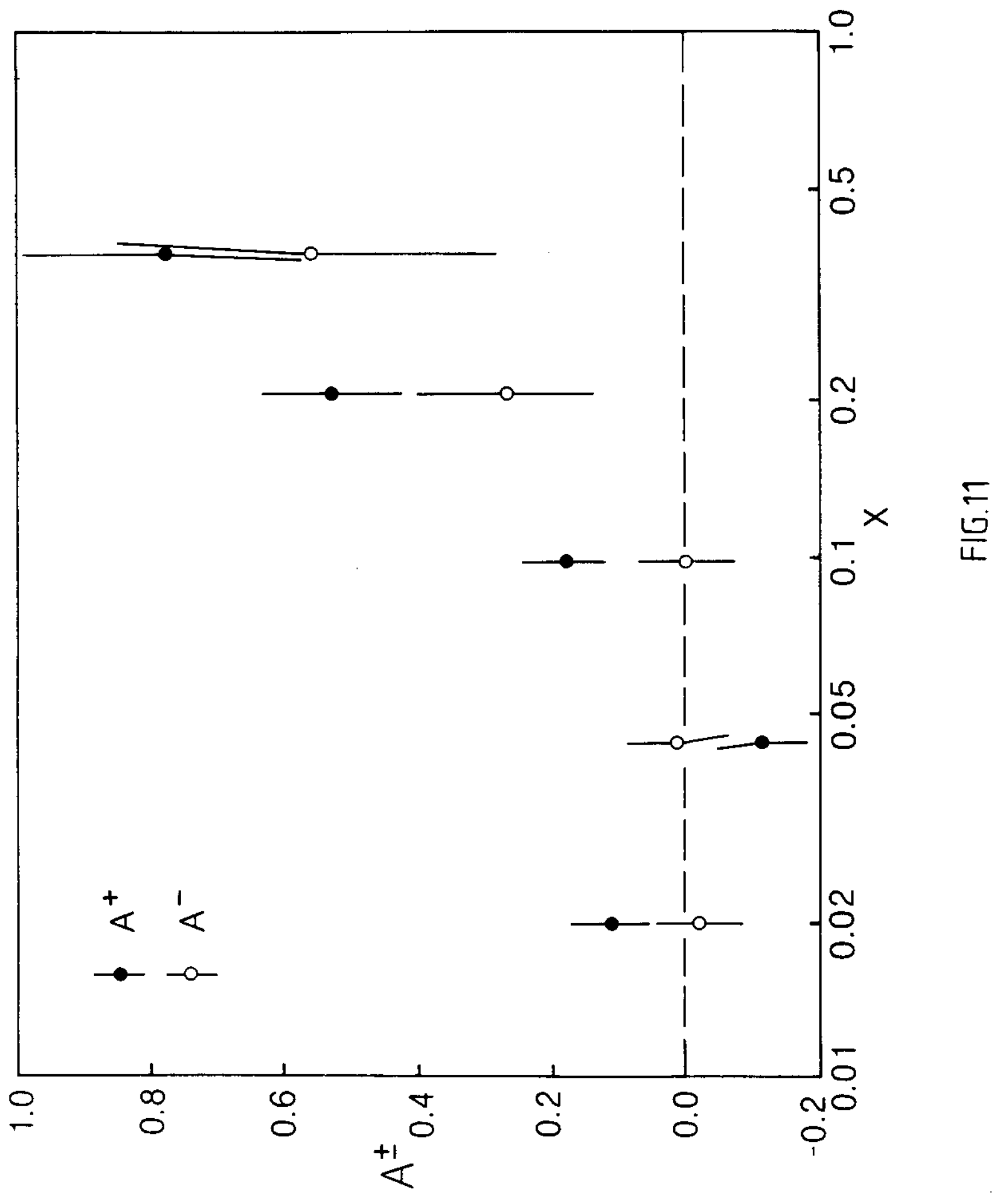




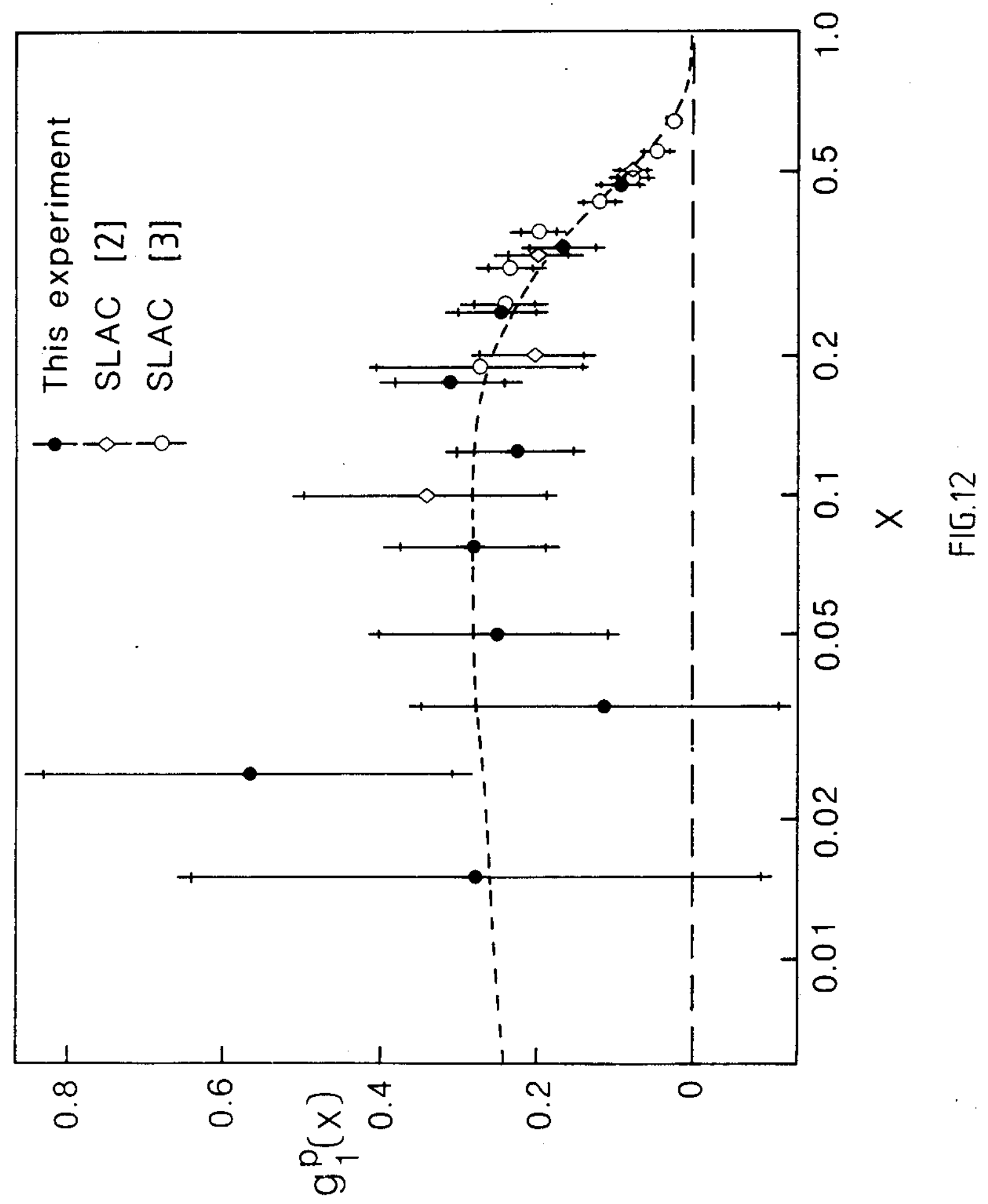




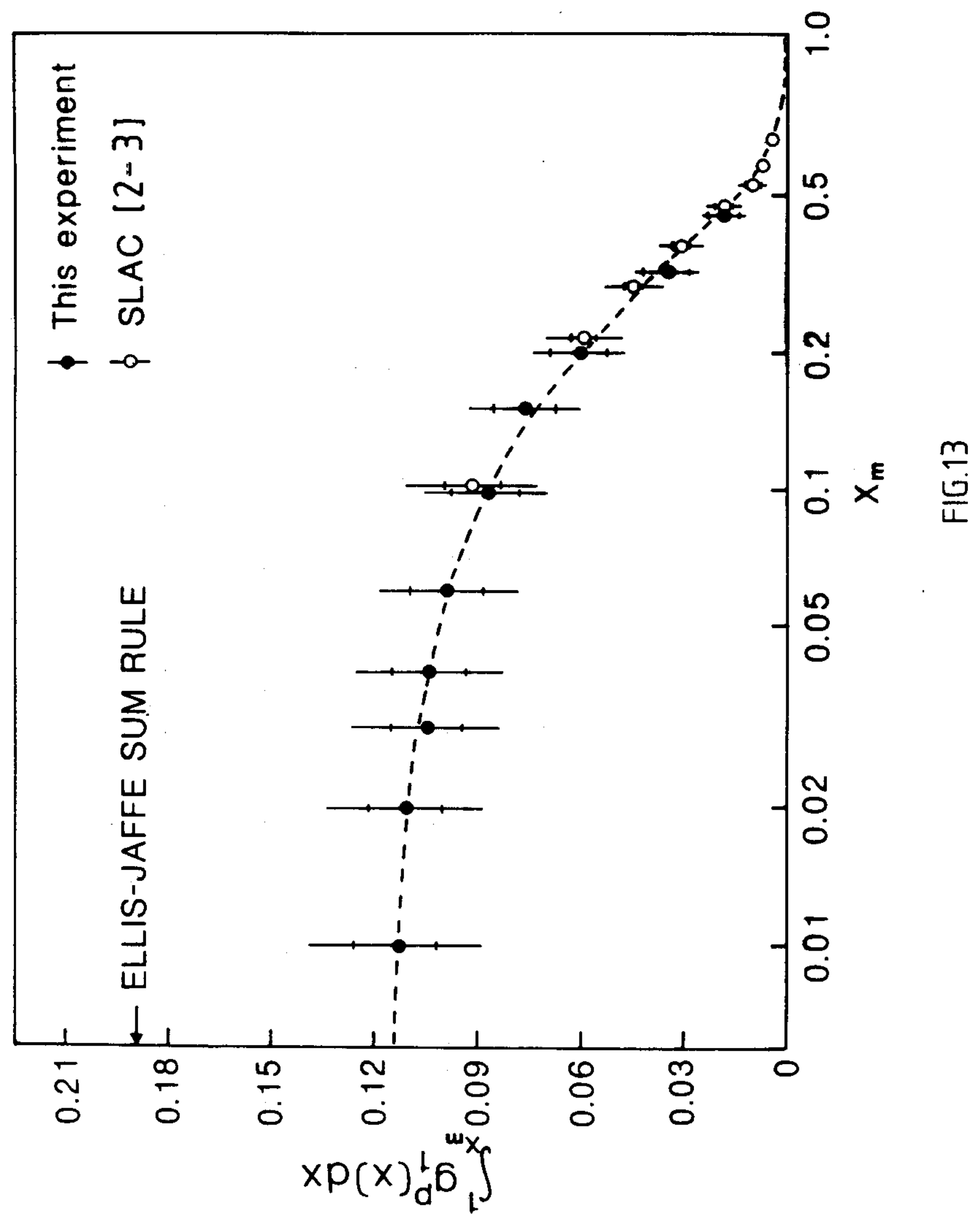

